\title{
RIGHT AND LEFT MODULES OVER THE FROBENIUS SKEW POLYNOMIAL RING IN THE F-FINITE CASE
}

\author{
RODNEY Y. SHARP AND YUJI YOSHINO
}

\begin{abstract}
The main purposes of this paper are to establish and exploit the result that, over a complete (Noetherian) local ring $R$ of prime characteristic for which the Frobenius homomorphism $f$ is finite, the appropriate restrictions of the Matlis-duality functor provide an equivalence between the category of left modules over the Frobenius skew polynomial ring $R[x, f]$ that are Artinian as $R$-modules and the category of right $R[x, f]$-modules that are Noetherian as $R$-modules.
\end{abstract}

\section{INTRODUCTION}

Throughout the paper, $R$ will denote a commutative Noetherian ring of prime characteristic $p$. We shall only assume that $R$ is local when this is explicitly stated; then, the notation ' $(R, \mathfrak{m})$ ' will denote that $\mathfrak{m}$ is the maximal ideal of $R$. We shall always denote by $f: R \longrightarrow R$ the Frobenius homomorphism, for which $f(r)=r^{p}$ for all $r \in R$. We shall work with the skew polynomial ring $R[x, f]$ associated to $R$ and $f$ in the indeterminate $x$ over $R$. Recall that $R[x, f]$ is, as a left $R$-module, freely generated by $\left(x^{i}\right)_{i \in \mathbb{N}_{0}}$ (we use $\mathbb{N}$ and $\mathbb{N}_{0}$ to denote the set of positive integers and the set of non-negative integers, respectively), and so consists of all polynomials $\sum_{i=0}^{n} r_{i} x^{i}$, where $n \in \mathbb{N}_{0}$ and $r_{0}, \ldots, r_{n} \in R$; however, its multiplication is subject to the rule

$$
x r=f(r) x=r^{p} x \quad \text { for all } r \in R .
$$

Note that $R[x, f]$ can be considered as a positively-graded ring $R[x, f]=\bigoplus_{n=0}^{\infty} R[x, f]_{n}$, with $R[x, f]_{n}=$ $R x^{n}$ for all $n \in \mathbb{N}_{0}$. The ring $R[x, f]$ will be referred to as the Frobenius skew polynomial ring over $R$.

In the case when $(R, \mathfrak{m})$ is local, several authors have used, often as an aid to the study of tight closure, the natural Frobenius action on the top local cohomology module $H_{\mathfrak{m}}^{\operatorname{dim} R}(R)$ of $R$ : see, for example, R. Fedder [5, Fedder and K.-i. Watanabe [6, K. E. Smith [23, N. Hara and Watanabe 8 and F. Enescu 2, 3]. The natural Frobenius action provides the top local cohomology module of $R$ with a natural structure as a left module over $R[x, f]$. The top local cohomology module of $R$ is Artinian as $R$-module, and so the papers cited above studied one example of a left $R[x, f]$-module that is Artinian as $R$-module. In recent years there have been studies of more general left $R[x, f]$-modules that are Artinian as $R$-modules: see, for example, M. Katzman [12] and the first author's [18, 20] and [21] (the authors are listed alphabetically).

On the other hand, the second author showed in [24, Proposition 3.5] that, if $R$ is $F$-finite, that is, the Frobenius map $f: R \longrightarrow R$ is a finite homomorphism, then each non-zero injective $R$-module $I$ has a non-trivial structure as a right $R[x, f]$-module. The main purpose of this paper is to build on that work to show that, when $R$ is $F$-finite, whenever $M$ is a left $R[x, f]$-module, then $\operatorname{Hom}_{R}(M, I)$ can be given a structure as right $R[x, f]$-module that extends its $R$-module structure, and, furthermore, whenever $N$ is a right $R[x, f]$-module, then $\operatorname{Hom}_{R}(N, I)$ can be given a structure as left $R[x, f]$-module that extends its $R$-module structure. Special attention is given to the case where $(R, \mathfrak{m})$ is local, complete and $F$ finite, and $I$ is taken to be $E:=E_{R}(R / \mathfrak{m})$, the injective envelope of the simple $R$-module. Classical

Date: October 25, 2018.

2000 Mathematics Subject Classification. Primary 13A35, 16S36, 13E05, 13E10, 13J10.

Key words and phrases. Commutative Noetherian ring, prime characteristic, Frobenius homomorphism, skew polynomial ring, Matlis duality.

The first author was partially supported by the Engineering and Physical Sciences Research Council of the United Kingdom (Overseas Travel Grant Number EP/C538803/1), and also by the Foundation for International Exchange Program of Okayama University. The second author was partially supported by Japan Society for the Promotion of Science (Grant-in-Aid (B) 21340008). 
Matlis duality yields that whenever $G$ is an $R$-module that is Artinian (respectively Noetherian), then the natural 'evaluation' $R$-homomorphism $G \longrightarrow \operatorname{Hom}_{R}\left(\operatorname{Hom}_{R}(G, E), E\right)$ is an isomorphism, and the 'Matlis dual' $\operatorname{Hom}_{R}(G, E)$ of $G$ is Noetherian (respectively Artinian). Our results, when combined with Matlis duality, lead to the conclusion that the appropriate restrictions of the functor $\operatorname{Hom}_{R}(-, E)$ provide an equivalence between the category of left $R[x, f]$-modules that are Artinian as $R$-modules (and all $R[x, f]$-homomorphisms between them) and the category of right $R[x, f]$-modules that are Noetherian as $R$-modules (and all $R[x, f]$-homomorphisms between them).

We can then use this equivalence to translate (in this complete, local, $F$-finite case) known results about left $R[x, f]$-modules that are Artinian as $R$-modules into results about right $R[x, f]$-modules that are Noetherian as $R$-modules. One example of this concerns the Hartshorne-Speiser-Lyubeznik Theorem, which we now recall.

0.1. Theorem (G. Lyubeznik [14, Proposition 4.4]). (Compare Hartshorne-Speiser [9, Proposition 1.11].) Suppose that $(R, \mathfrak{m})$ is local, and let $G$ be a left $R[x, f]$-module that is Artinian as $R$-module. Then there exists $e \in \mathbb{N}_{0}$ with the following property: whenever $g \in G$ is such that $x^{n} g=0$ for some $n \in \mathbb{N}$, then $x^{e} g=0$.

Hartshorne and Speiser first proved this result in the case where $R$ is local and contains its residue field which is perfect. Lyubeznik applied his theory of $F$-modules to obtain the result without restriction on the local ring $R$ of characteristic $p$. There is a short proof of the Hartshorne-Speiser-Lyubeznik Theorem in [19]. It was shown in [17, Corollary 1.8] that the result is still valid if the hypothesis that $R$ be local is dropped.

The Hartshorne-Speiser-Lyubeznik Theorem has been used to establish the existence of uniform test exponents for Frobenius closures of parameter ideals in local rings in certain circumstances. Let $\mathfrak{a}$ be an ideal of $R$; let $n \in \mathbb{N}_{0}$. Recall that the $n$-th Frobenius power $\mathfrak{a}^{\left[p^{n}\right]}$ of $\mathfrak{a}$ is the ideal of $R$ generated by all $p^{n}$-th powers of elements of $\mathfrak{a}$. The Frobenius closure $\mathfrak{a}^{F}$ of $\mathfrak{a}$ is defined by

$$
\mathfrak{a}^{F}:=\left\{r \in R \mid \text { there exists } n \in \mathbb{N}_{0} \text { such that } r^{p^{n}} \in \mathfrak{a}^{\left[p^{n}\right]}\right\} .
$$

This is an ideal of $R$, and so is finitely generated; therefore there exists a power $Q_{0}$ of $p$ such that $\left(\mathfrak{a}^{F}\right)^{\left[Q_{0}\right]}=\mathfrak{a}^{\left[Q_{0}\right]}$, and we define $Q(\mathfrak{a})$ to be the smallest power of $p$ with this property. In [13, Theorem 2.5], M. Katzman and Sharp used the Hartshorne-Speiser-Lyubeznik Theorem to show that, when $(R, \mathfrak{m})$ is local and Cohen-Macaulay, the set

$$
\{Q(\mathfrak{a}): \mathfrak{a} \text { is an ideal generated by part of a system of parameters of } R\}
$$

is bounded; in [11, C. Huneke, Katzman, Sharp and Y. Yao again used the Hartshorne-SpeiserLyubeznik Theorem (and quite a few other techniques) to establish the same conclusion in a generalized Cohen-Macaulay local ring.

We are able to use our above-mentioned equivalence of categories to prove the following result (as Theorem 3.1), in the case where $R$ is $F$-finite, local and complete.

Theorem. Assume that $(R, \mathfrak{m})$ is $F$-finite, local and complete. Let $N$ be a right $R[x, f]$-module that is Noetherian as $R$-module. Then there exists $e \in \mathbb{N}_{0}$ such that $N x^{e}=N x^{e+1}$.

This result can be viewed as a dual of the Hartshorne-Speiser-Lyubeznik Theorem. A natural question is whether this 'dual Hartshorne-Speiser-Lyubeznik Theorem' is still valid if all the hypotheses about $R$, except the one that it (is a commutative Noetherian ring and) has characteristic $p$, are dropped: we shall show, in the final section of the paper, that this question has an affirmative answer.

Another useful result about left $R[x, f]$-modules that are Artinian as $R$-modules concerns graded annihilators: the graded annihilator of a (left or right) $R[x, f]$-module $T$ is the largest graded two-sided ideal of $R[x, f]$ that annihilates $T$.

0.2. Theorem (R. Y. Sharp [18, Corollary 3.11]). Let $G$ be a left $R[x, f]$-module that is Artinian as $R$-module. Suppose that $G$ is $x$-torsion-free, that is, $x g=0$ for $g \in G$ implies that $g=0$. Then there are only finitely many graded annihilators of $R[x, f]$-submodules of $G$.

The first author has been able to use this result to prove existence theorems about tight closure test elements: see [21, Theorem 4.16]. 
We are able to use our above-mentioned equivalence of categories to prove the following result (as Theorem 3.5), in the case where $R$ is $F$-finite, local and complete.

Theorem. Assume that $(R, \mathfrak{m})$ is $F$-finite, local and complete. Let $M$ be a right $R[x, f]$-module that is Noetherian as $R$-module. Suppose that $M$ is $x$-divisible, that is $M=M x$. Then there are only finitely many graded annihilators of $R[x, f]$-homomorphic images of $M$.

Again, it is natural to ask whether this result is still valid if all the hypotheses about $R$, except the one that it (is a commutative Noetherian ring and) has characteristic $p$, are dropped. At the time of writing, we have not been able to answer to this question.

Note. Most of the research reported in this paper was carried out during a visit by Sharp to the University of Okayama in March 2008. After the paper had been accepted, it was pointed out to us that some of its results have been independently obtained by M. Blickle and G. Boeckle in their paper [1. In detail, Theorem 1.20 below appears in [1, Section 5.1], and the result of Theorem 3.4 below follows from [1, Proposition 2.14] (which Blickle and Boeckle prove via an argument of O. Gabber from [7, Section 13]).

\section{Right And Left modules over the Frobenius SkeW POlynomial Ring}

The notation and terminology used in the Introduction will be used throughout the paper.

First of all, let us recall some of the basic facts about bimodules, which we shall use in the rest of the paper. See, for example, Rotman [16, Lemma 8.80, Theorem 8.99].

1.1. Remark. Let $A, B, C$ and $D$ be commutative rings.

(i) An Abelian group $M$ is an $(A, B)$-bimodule if $M$ is a left $A$-module, a right $B$-module and the two actions of the rings are related by the following rule:

$$
(a m) b=a(m b) \quad \text { for all } a \in A, b \in B \text { and } m \in M \text {. }
$$

(ii) If $M$ is an $(A, B)$-bimodule and $N$ is a $(B, C)$-bimodule, then $M \otimes_{B} N$ is naturally an $(A, C)$ bimodule, where the bimodule structure is given by

$$
a(m \otimes n) c=(a m) \otimes(n c) \quad \text { for all } a \in A, c \in C, m \in M \text { and } n \in N .
$$

(iii) If $M$ is an $(A, B)$-bimodule and $N$ is an $(A, C)$-bimodule, then the set of all left $A$-homomorphisms from $M$ to $N$, denoted by $\operatorname{Hom}_{l A}(M, N)$, is naturally a $(B, C)$-bimodule, where

$$
(b \varphi c)(m)=(\varphi(m b)) c \text { for all } b \in B, c \in C, m \in M \text { and } \varphi \in \operatorname{Hom}_{l A}(M, N) .
$$

Similarly if $M$ is an ( $A, B)$-bimodule and $N$ is a $(C, B)$-bimodule, then the set of all right $B$-homomorphisms from $M$ to $N$, denoted by $\operatorname{Hom}_{r B}(M, N)$, is naturally a $(C, A)$-bimodule, where

$$
(c \psi a)(m)=c(\psi(a m)) \quad \text { for all } c \in C, a \in A, m \in M \text { and } \psi \in \operatorname{Hom}_{r B}(M, N) .
$$

(iv) If $M$ is an $(A, B)$-bimodule, $N$ is a $(B, C)$-bimodule, and $L$ is an $(A, D)$-bimodule, then there exists a $(C, D)$-bimodule isomorphism, the so-called adjoint isomorphism,

$$
\Xi: \operatorname{Hom}_{l A}\left(M \otimes_{B} N, L\right) \stackrel{\cong}{\longrightarrow} \operatorname{Hom}_{l B}\left(N, \operatorname{Hom}_{l A}(M, L)\right)
$$

which is such that

$$
((\Xi(\phi))(n))(m)=\phi(m \otimes n) \quad \text { for all } n \in N, m \in M \text { and } \phi \in \operatorname{Hom}_{l A}\left(M \otimes_{B} N, L\right) .
$$

(v) Similarly, if $M$ is an $(A, B)$-bimodule, $N$ is a $(B, C)$-bimodule, and $L$ is a $(D, C)$-bimodule, then there exists an 'adjoint' $(D, A)$-bimodule isomorphism

$$
\Theta: \operatorname{Hom}_{r C}\left(M \otimes_{B} N, L\right) \stackrel{\cong}{\longrightarrow} \operatorname{Hom}_{r B}\left(M, \operatorname{Hom}_{r}(N, L)\right)
$$

which is such that

$$
((\Theta(\varphi))(m))(n)=\varphi(m \otimes n) \quad \text { for all } m \in M, n \in N \text { and } \varphi \in \operatorname{Hom}_{r C}\left(M \otimes_{B} N, L\right) .
$$


(vi) If $M$ is an $(A, B)$-bimodule, $N$ is a $(C, D)$-bimodule, and $L$ is an $(A, D)$-bimodule, then there exists a $(B, C)$-bimodule isomorphism

$$
\Omega: \operatorname{Hom}_{l A}\left(M, \operatorname{Hom}_{r D}(N, L)\right) \cong \operatorname{Hom}_{r D}\left(N, \operatorname{Hom}_{l A}(M, L)\right)
$$

for which

$((\Omega(\psi))(n))(m)=(\psi(m))(n) \quad$ for all $m \in M, n \in N$ and $\psi \in \operatorname{Hom}_{l A}\left(M, \operatorname{Hom}_{r D}(N, L)\right)$.

Recall that $R$ denotes a commutative Noetherian ring of prime characteristic $p$ and that $f: R \longrightarrow R$ denotes the Frobenius homomorphism. We shall only assume that $R$ is $F$-finite when this is explicitly stated.

Let $M$ be an $R$-module. We always regard $M$ as an $(R, R)$-bimodule by $r \cdot m \cdot s=r s m$ for $r, s \in R$ and $m \in M$. On the other hand, we define the $(R, R)$-bimodule $M_{f}$ to be $M_{f}=M$ as Abelian group with $(R, R)$-bimodule structure defined by

$$
r \cdot m \cdot s=r s^{p} m \text { for all } r, s \in R \text { and } m \in M .
$$

Note that the Frobenius map $f: R \rightarrow R_{f}$ is a right $R$-module homomorphism.

Similarly, we define the $(R, R)$-bimodule ${ }_{f} M$ to be ${ }_{f} M=M$ as Abelian group with $(R, R)$-bimodule structure defined by

$$
r \cdot m \cdot s=r^{p} s m \text { for all } r, s \in R \text { and } m \in M \text {. }
$$

1.2. Remark. Let $M$ be an $R$-module.

(i) By 1.1(ii), $M \otimes_{R} R_{f}$ has naturally a structure of $(R, R)$-bimodule. The action of $R$ is given by

$$
s \cdot(m \otimes r) \cdot s^{\prime}=s r s^{\prime p} m \otimes 1 \quad \text { for all } r, s, s^{\prime} \in R \text { and } m \in M .
$$

Thus there is an isomorphism $M_{f} \cong M \otimes_{R} R_{f}$ as $(R, R)$-bimodules.

(ii) Similarly, ${ }_{f} R \otimes_{R} M$ is an $(R, R)$-bimodule with action given by

$$
s \cdot(r \otimes m) \cdot s^{\prime}=1 \otimes s^{p} r s^{\prime} m \quad \text { for all } r, s, s^{\prime} \in R \text { and } m \in M .
$$

There is an isomorphism ${ }_{f} M \cong{ }_{f} R \otimes_{R} M$ of $(R, R)$-bimodules.

(iii) By 1.1(iii), the Abelian group $\operatorname{Hom}_{l R}\left(R_{f}, M\right)$ consisting of all left $R$-homomorphisms from $R_{f}$ to $M$ is an $(R, R)$-bimodule with action of $R$ given by

$$
\left(s \varphi s^{\prime}\right)(r)=(\varphi(r \cdot s)) s^{\prime}=r s^{p} s^{\prime} \varphi(1) \quad \text { for all } r, s, s^{\prime} \in R \text { and } \varphi \in \operatorname{Hom}_{l R}\left(R_{f}, M\right) .
$$

It is easy to see that $\operatorname{Hom}_{l R}\left(R_{f}, M\right) \cong{ }_{f} M$ as $(R, R)$-bimodules.

(iv) Similarly, the set $\operatorname{Hom}_{r R}\left(R_{f}, M\right)$ of all right $R$-homomorphisms from $R_{f}$ to $M$ is an $(R, R)$ bimodule with

$$
\left(s \psi s^{\prime}\right)(r)=s \psi\left(s^{\prime} r\right) \quad \text { for all } r, s, s^{\prime} \in R \text { and } \psi \in \operatorname{Hom}_{r R}\left(R_{f}, M\right) .
$$

We shall use a refinement of the following result.

1.3. Lemma (Y. Yoshino [24, Lemma 3.6]). Suppose that $(R, \mathfrak{m})$ is local and F-finite. Denote by $E$ the injective envelope $E_{R}(R / \mathfrak{m})$ of the simple $R$-module, which we regard as a right $R$-module. Then there is a right $R$-module isomorphism $E \stackrel{\cong}{\longrightarrow} \operatorname{Hom}_{r R}\left(R_{f}, E\right)$, where $\operatorname{Hom}_{r R}\left(R_{f}, E\right)$ carries the right $R$-module structure described in Remark 1.2(iv).

We shall use the following refinement, in which it is not assumed that $R$ is local.

1.4. Lemma. Suppose that $R$ is $F$-finite, and let $I$ be an injective $R$-module. Then there is an $(R, R)$ bimodule isomorphism

$$
\operatorname{Hom}_{l R}\left(R_{f}, I\right) \stackrel{\cong}{\longrightarrow} \operatorname{Hom}_{r R}\left(R_{f}, I\right) .
$$

Proof. It is a consequence of the adjoint isomorphism of Remark 1.1(v) that $\operatorname{Hom}_{r}\left(R_{f}, I\right)$ is injective as right $R$-module. On the other hand, by 1.2(iii), we have an isomorphism of $(R, R)$-bimodules $\operatorname{Hom}_{l R}\left(R_{f}, I\right) \cong{ }_{f} I$.

We can use the well-known decomposition theory for injective $R$-modules due to E. Matlis (reviewed in, for example, [15, §18]) to see that it is enough for us to prove the result when $I=E_{R}(R / \mathfrak{p})$ for a prime ideal $\mathfrak{p}$ of $R$, and so we assume that this is so in the rest of the proof. 
Since, for a prime ideal $\mathfrak{q}$ of $R$, each element of $E_{R}(R / \mathfrak{q})$ is annihilated by some power of $\mathfrak{q}$, and multiplication by an element $r \in R \backslash \mathfrak{q}$ provides an automorphism of $E_{R}(R / \mathfrak{q})$, it follows that $\operatorname{Hom}_{r R}\left(R_{f}, E_{R}(R / \mathfrak{p})\right.$ ) (with the right $R$-module structure described in Remark 1.2(iv)) is isomorphic to a direct sum of $\mu$ copies of $E_{R}(R / \mathfrak{p})$. First we prove that the cardinal $\mu$ is exactly 1 . Thus

$$
\operatorname{Hom}_{r R}\left(R_{f}, E_{R}(R / \mathfrak{p})\right) \cong \bigoplus \mu\left(E_{R}(R / \mathfrak{p})\right)
$$

as right $R$-modules. We consider $\left(R_{f}\right)_{\mathfrak{p}}$ as the localization of the right $R$-module $R_{f}$ at $\mathfrak{p}$ and write the resulting action of $R_{\mathfrak{p}}$ on the right. (Thus $(r / s) \cdot(a / t)=r a^{p} / s t$ for $r \in R_{f}, a \in R$ and $s, t \in R \backslash \mathfrak{p}$.) We can also endow this $\left(R_{f}\right)_{\mathfrak{p}}$ with a left $R_{\mathfrak{p}}$-module structure under which

$$
\left(\frac{a}{t}\right) \cdot\left(\frac{r}{s}\right)=\frac{t^{p-1} a r}{s t} \quad \text { for all } r \in R_{f}, a \in R \text { and } s, t \in R \backslash \mathfrak{p} .
$$

These two structures turn $\left(R_{f}\right)_{\mathfrak{p}}$ into an $\left(R_{\mathfrak{p}}, R_{\mathfrak{p}}\right)$-bimodule, and then there is an $\left(R_{\mathfrak{p}}, R_{\mathfrak{p}}\right)$-bimodule isomorphism $\beta:\left(R_{f}\right)_{\mathfrak{p}} \stackrel{\cong}{\longrightarrow}\left(R_{\mathfrak{p}}\right)_{f}$ for which $\beta(r / s)=r / s^{p}$ for all $r \in R_{f}$ and $s \in R \backslash \mathfrak{p}$. Since $R_{f}$ is finitely generated as right $R$-module, there is a right $R_{\mathfrak{p}}$-module isomorphism

$$
\left(\operatorname{Hom}_{r R}\left(R_{f}, E_{R}(R / \mathfrak{p})\right)\right)_{\mathfrak{p}} \cong \operatorname{Hom}_{r R_{\mathfrak{p}}}\left(\left(R_{f}\right)_{\mathfrak{p}},\left(E_{R}(R / \mathfrak{p})\right)_{\mathfrak{p}}\right)
$$

when $\operatorname{Hom}_{r R}\left(R_{f}, E_{R}(R / \mathfrak{p})\right.$ ) (respectively $\left.\operatorname{Hom}_{r R_{\mathfrak{p}}}\left(\left(R_{f}\right)_{\mathfrak{p}},\left(E_{R}(R / \mathfrak{p})\right)_{\mathfrak{p}}\right)\right)$ is considered as a right $R$ module (respectively a right $R_{\mathfrak{p}}$-module) via Remark 1.1(iii). One can use this isomorphism, and the isomorphism $\beta$ above, to see that there is a right $R_{\mathfrak{p}}$-module isomorphism

$$
\left(\operatorname{Hom}_{r R}\left(R_{f}, E_{R}(R / \mathfrak{p})\right)\right)_{\mathfrak{p}} \cong \operatorname{Hom}_{r R_{\mathfrak{p}}}\left(\left(R_{\mathfrak{p}}\right)_{f}, E_{R_{\mathfrak{p}}}\left(R_{\mathfrak{p}} / \mathfrak{p} R_{\mathfrak{p}}\right)\right) .
$$

The last module is right $R_{\mathfrak{p}}$-isomorphic to $E_{R_{\mathfrak{p}}}\left(R_{\mathfrak{p}} / \mathfrak{p} R_{\mathfrak{p}}\right)$ by Lemma 1.3 Therefore $\mu=1$.

We have thus shown that there is a right $R$-module isomorphism $\varphi: I \rightarrow \operatorname{Hom}_{r R}\left(R_{f}, I\right)$. To finish the proof, we show that this mapping $\varphi$, regarded as a mapping ${ }_{f} I \rightarrow \operatorname{Hom}_{r R}\left(R_{f}, I\right)$, is actually a left $R$-module homomorphism, and therefore an $(R, R)$-bimodule isomorphism. For $z \in{ }_{f} I$ and $a \in R$, we have, for all $r \in R_{f}$,

$$
\begin{aligned}
\varphi(a \cdot z)(r) & =\varphi\left(z a^{p}\right)(r)=\left(\varphi(z) a^{p}\right)(r)=\varphi(z)\left(a^{p} r\right) \\
& =\varphi(z)(r \cdot a)=(\varphi(z)(r)) a=a(\varphi(z)(r))=(a \varphi(z))(r),
\end{aligned}
$$

so that $\varphi(a \cdot z)=a \varphi(z)$. Therefore $\varphi$ is a left $R$-homomorphism.

1.5. Remark. If, in Lemma 1.4 we drop the hypothesis that $R$ is $F$-finite, then the conclusion is no longer always true. For one example, let $K$ be a countable field of characteristic $p$ with $\left[K: K^{p}\right]$ infinite but countable, and set $R=K$. We show now that $\operatorname{Hom}_{r K}\left(K_{f}, K\right) ¥_{f} K$ as right $K$-modules. Assume that $\operatorname{Hom}_{r K}\left(K_{f}, K\right) \cong{ }_{f} K$ as right $K$-modules and seek a contradiction.

Let $\bar{K}$ be an algebraic closure of $K$, and let $K^{1 / p}$ denote the subfield of $\bar{K}$ consisting of all $p$ th roots of elements of $K$. The assumption implies that $\operatorname{Hom}_{K}\left(K^{1 / p}, K\right) \cong K^{1 / p}$ as $K^{1 / p}$-modules. In particular, $\operatorname{Hom}_{K}\left(K^{1 / p}, K\right)$ has countable dimension as a vector space over $K$. Let $\left(\alpha_{n}\right)_{n \in \mathbb{N}}$ be a $K$-basis of $K^{1 / p}$, so that $K^{1 / p}=\bigoplus_{n \in \mathbb{N}} K \alpha_{n}$. Then $\operatorname{Hom}_{K}\left(K^{1 / p}, K\right)=\operatorname{Hom}_{K}\left(\bigoplus_{n \in \mathbb{N}} K \alpha_{n}, K\right) \cong \prod_{n \in \mathbb{N}} \operatorname{Hom}_{K}\left(K \alpha_{n}, K\right)$, and this has uncountable dimension as a vector space over $K$, and this is a contradiction.

1.6. Discussion. The Frobenius skew polynomial ring $R[x, f]$ was defined in the Introduction. It follows from [13, Lemma 1.3] that extension of the $R$-module structure on an $R$-module $H$ to a structure of left $R[x, f]$-module is equivalent to the provision of an Abelian group homomorphism $\xi: H \longrightarrow H$ for which $\xi(r h)=r^{p} \xi(h)$ for all $r \in R$ and $h \in H$. (In fact, $\xi$ and the action of $x$ are related by the formula $\xi(h)=x h$ for all $h \in H$.)

There is a bijective correspondence between $\operatorname{Hom}_{l R}\left(R_{f} \otimes_{R} H, H\right)$ and

$$
\left\{\xi \in \operatorname{End}_{\mathbb{Z}}(H) \mid \xi(r h)=r^{p} \xi(h) \text { for all } r \in R \text { and } h \in H\right\}
$$

under which $\alpha \in \operatorname{Hom}_{l R}\left(R_{f} \otimes_{R} H, H\right)$ corresponds to $h \mapsto \alpha(1 \otimes h)$. In view of this, we are going to use the notation $(H, \alpha)$ to describe a left $R[x, f]$-module $\mathbf{H}$, where $H$ is the underlying $R$-module and $\alpha \in \operatorname{Hom}_{l R}\left(R_{f} \otimes_{R} H, H\right)$ is such that $x h=\alpha(1 \otimes h)$ for all $h \in H$.

Under the adjoint isomorphism of Remark 1.1(iv), an $\alpha \in \operatorname{Hom}_{l R}\left(R_{f} \otimes_{R} H, H\right)$ corresponds to an $\widetilde{\alpha} \in \operatorname{Hom}_{l R}\left(H, \operatorname{Hom}_{l R}\left(R_{f}, H\right)\right)$. Note that $x h=(\widetilde{\alpha}(h))(1)$ for all $h \in H$. We write $\mathbf{H}=(H, \alpha)=[H, \widetilde{\alpha}]$. 
With such notation, a left $R[x, f]$-homomorphism $\mathbf{H}=(H, \alpha) \rightarrow \mathbf{H}^{\prime}=\left(H^{\prime}, \alpha^{\prime}\right)$ of left $R[x, f]$-modules is an $R$-homomorphism $\varphi: H \rightarrow H^{\prime}$ for which the diagram

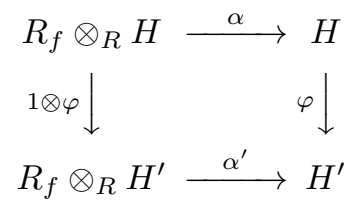

commutes.

1.7. Discussion. Similarly, extension of the $R$-module structure on an $R$-module $M$ to a structure of right $R[x, f]$-module is equivalent to the provision of an Abelian group homomorphism $\xi: M \longrightarrow M$ for which $\xi\left(m r^{p}\right)=\xi(m) r$ for all $r \in R$ and $m \in M$. The map $\xi$ and the action of $x$ are related by the formula $\xi(m)=m x$ for all $m \in M$.

There is a bijective correspondence between $\operatorname{Hom}_{r}\left(M \otimes_{R} R_{f}, M\right)$ and

$$
\left\{\xi \in \operatorname{End}_{\mathbb{Z}}(M) \mid \xi\left(m r^{p}\right)=\xi(m) r \text { for all } r \in R \text { and } m \in M\right\}
$$

under which $\beta \in \operatorname{Hom}_{r R}\left(M \otimes_{R} R_{f}, M\right)$ corresponds to $m \mapsto \beta(m \otimes 1)$. In view of this, we are going to use the notation $(M, \beta)$ to describe a right $R[x, f]$-module $\mathbf{M}$, where $M$ is the underlying $R$-module and $\beta \in \operatorname{Hom}_{r R}\left(M \otimes_{R} R_{f}, M\right)$ is such that $m x=\beta(m \otimes 1)$ for all $m \in M$.

Under the adjoint isomorphism of Remark 1.1(v), a $\beta \in \operatorname{Hom}_{r R}\left(M \otimes_{R} R_{f}, M\right)$ corresponds to a $\widetilde{\beta} \in \operatorname{Hom}_{r R}\left(M, \operatorname{Hom}_{r R}\left(R_{f}, M\right)\right)$. Note that $m x=(\widetilde{\beta}(m))(1)$ for all $m \in M$. We write $\mathbf{M}=(M, \beta)=$ $[M, \widetilde{\beta}]$.

1.8. Notation. We shall use $R[x, f]$ Mod to denote the category of all left $R[x, f]$-modules and left $R[x, f]$ homomorphisms between them, and $\operatorname{Mod}_{R[x, f]}$ to denote the category of all right $R[x, f]$-modules and right $R[x, f]$-homomorphisms between them.

1.9. Examples. (i) The Frobenius endomorphism $f: R \rightarrow R$ induces a left $R$-module homomorphism $\alpha: R_{f} \otimes_{R} R \rightarrow R$ for which $\alpha(a \otimes b)=a f(b)=a b^{p}$ for all $a \in R_{f}$ and $b \in R$. This therefore yields the left $R[x, f]$-module $(R, \alpha)$, in which we have $x r=r^{p}$ for all $r \in R$.

Let $c \in R$ be any element. Then there is a left $R$-module homomorphism $\alpha_{c}: R_{f} \otimes R \rightarrow R$ such that $\alpha_{c}(a \otimes b)=c a b^{p}$ for $a \in R_{f}$ and $b \in R$. Thus we obtain a left $R[x, f]$-module $\left(R, \alpha_{c}\right)$, in which $x r=c r^{p}$ for all $r \in R$. It is straightforward to check that $(R, \alpha) \cong\left(R, \alpha_{c}\right)$ as left $R[x, f]$-modules if and only if $c$ is a unit in $R$ possessing a $(p-1)$ th root in $R$. Thus it is possible for there to be many left $R[x, f]$-modules with the same underlying $R$-module.

(ii) Suppose that our ring $R$ is reduced and that we are given a non-trivial $R^{p}$-homomorphism $\pi: R \rightarrow R^{p}$. (In the case where $R$ is $F$-finite and $F$-pure, we can find such a $\pi$ that is a surjective mapping, because $R^{p}$ is a direct summand of $R$ as an $R^{p}$-module: see [10, Corollary 5.3].) In this situation, we have a right $R$-module homomorphism $\beta: R \otimes_{R} R_{f} \rightarrow R$ for which $\beta(a \otimes b)=\pi(a b)^{1 / p}$ for all $a \in R$ and $b \in R_{f}$. This yields a right $R[x, f]$-module $(R, \beta)$, in which we have $r x=\pi(r)^{1 / p}$ for all $r \in R$.

We have shown in 1.4 that whenever $R$ is $F$-finite and $I$ is an injective $R$-module, there is an $(R, R)$-bimodule isomorphism $\Psi:{ }_{f} I \rightarrow \operatorname{Hom}_{r R}\left(R_{f}, I\right)$; of course, $\Psi$ is, in particular, a right $R$-module homomorphism. Therefore we have the following as a corollary to 1.4

1.10. Corollary. Suppose that $R$ is $F$-finite, and let $I$ be an injective $R$-module. Then there is a right $R[x, f]$-module $\mathbf{I}=[I, \Psi]$ which has $I$ as underlying $R$-module, and is such that $\Psi:{ }_{f} I \rightarrow \operatorname{Hom}_{r}\left(R_{f}, I\right)$ is an $(R, R)$-bimodule isomorphism. Note that $z x=(\Psi(z))(1)$ for all $z \in I$.

1.11. Lemma. Let the situation and notation be as in Corollary 1.10 , and consider the right $R[x, f]$ module $\mathbf{I}=[I, \Psi]$. Then $\mathbf{I}$ has the following property: if $z \in \mathbf{I}$ is such that, for a fixed $n \in \mathbb{N}_{0}$, we have $z r x^{n}=0$ for all $r \in R$, then $z=0$.

Proof. The claim is clear when $n=0$, and we deal now with the case where $n=1$. We have

$$
0=(z r) x=(\Psi(z r))(1)=((\Psi(z)) r)(1)=(\Psi(z))(r) \quad \text { for all } r \in R .
$$


Therefore $\Psi(z)=0$, so that $z=0$ because $\Psi$ is an isomorphism.

Now suppose, inductively, that $n \in \mathbb{N}$ with $n>1$, and that the claim has been proved for all smaller values of $n$. Suppose that $z r x^{n}=0$ for all $r \in R$. Then $\left(z r x^{n-1}\right) s x=z r s^{p^{n-1}} x^{n}=0$ for all $r, s \in R$. It follows from the case where $n=1$ that $z r x^{n-1}=0$ for all $r \in R$; it then follows from the inductive hypothesis that $z=0$.

1.12. Discussion. Throughout the rest of this section, assume that our $\operatorname{ring} R$ is $F$-finite, and let $I$ be an injective $R$-module. We fix a right $R[x, f]$-module structure on $I$ as in Corollary 1.10 , so that $\mathbf{I}=[I, \Psi]$ is a right $R[x, f]$-module with $\Psi:{ }_{f} I \rightarrow \operatorname{Hom}_{r}\left(R_{f}, I\right)$ an $(R, R)$-bimodule isomorphism. We denote by $(-)^{\vee}$ the duality functor determined by $I$, so that $X^{\vee}=\operatorname{Hom}_{R}(X, I)$ for each $R$-module $X$.

Now suppose we are given a left $R[x, f]$-module $\mathbf{H}=(H, \alpha)$ with $\alpha \in \operatorname{Hom}_{l R}\left(R_{f} \otimes_{R} H, H\right)$.

(i) Here we produce a right $R[x, f]$-module structure on $H^{\vee}$.

First apply the functor $(-)^{\vee}$ to the left $R$-homomorphism $\alpha: R_{f} \otimes_{R} H \rightarrow H$ : the result is a right $R$-homomorphism $\alpha^{\vee}: H^{\vee} \rightarrow \operatorname{Hom}_{l R}\left(R_{f} \otimes_{R} H, I\right)$. But there is an $(R, R)$-bimodule isomorphism $\operatorname{Hom}_{l R}\left(R_{f} \otimes_{R} H, I\right) \stackrel{\cong}{\longrightarrow} \operatorname{Hom}_{l R}\left(H, \operatorname{Hom}_{l R}\left(R_{f}, I\right)\right)$ given by Remark 1.1(iv), and use of the $(R, R)$-bimodule isomorphism $\Psi$ produces a further $(R, R)$-bimodule isomorphism

$$
\operatorname{Hom}_{l R}\left(H, \operatorname{Hom}_{l R}\left(R_{f}, I\right)\right) \stackrel{\cong}{\longrightarrow} \operatorname{Hom}_{l R}\left(H, \operatorname{Hom}_{r}\left(R_{f}, I\right)\right) .
$$

In addition, Remark 1.1)(vi) provides an $(R, R)$-bimodule isomorphism

$$
\operatorname{Hom}_{l R}\left(H, \operatorname{Hom}_{r R}\left(R_{f}, I\right)\right) \stackrel{\cong}{\longrightarrow} \operatorname{Hom}_{r R}\left(R_{f}, \operatorname{Hom}_{l R}(H, I)\right) \text {. }
$$

Composition of these therefore yields a right $R$-homomorphism $\gamma: H^{\vee} \longrightarrow \operatorname{Hom}_{r}\left(R_{f}, H^{\vee}\right)$, and we shall denote by $D(\alpha)$ the right $R$-homomorphism $H^{\vee} \otimes_{R} R_{f} \rightarrow H^{\vee}$ that corresponds to $\gamma$ under the adjoint isomorphism of Remark 1.1)(v). (Note that $H^{\vee}=\operatorname{Hom}_{l R}(H, I)=$ $\operatorname{Hom}_{r R}(H, I)$.)

Thus $D(\alpha)$ makes $H^{\vee}$ into a right $R[x, f]$-module. We define

$$
\mathbf{D}(\mathbf{H})=\mathbf{D}(H, \alpha):=\left(H^{\vee}, D(\alpha)\right)=\left[H^{\vee}, \gamma\right] .
$$

It is straightforward to use the above definition of $\gamma$ to check that

$$
(D(\alpha)(m \otimes r))(h)=(\Psi(m(\alpha(1 \otimes h))))(r) \quad \text { for all } m \in H^{\vee}, r \in R_{f} \text { and } h \in H .
$$

(ii) Now let $\mathbf{H}^{\prime}=\left(H^{\prime}, \alpha^{\prime}\right)$ be a second left $R[x, f]$-module and let $\varphi: \mathbf{H} \rightarrow \mathbf{H}^{\prime}$ be a left $R[x, f]$ homomorphism. Thus $\varphi$ is an $R$-homomorphism $H \rightarrow H^{\prime}$ which makes the diagram

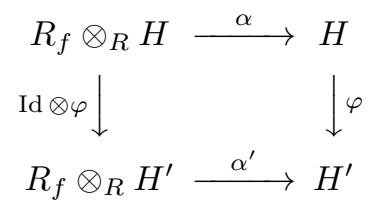

commute. It is straightforward to check that the diagram

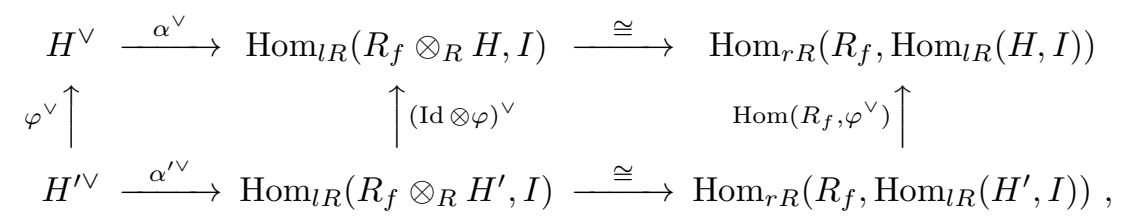

in which the upper horizontal isomorphism is the one used in the construction in part (i) and the lower horizontal isomorphism is the corresponding one for $\mathbf{H}^{\prime}$, commutes. Therefore $\varphi^{\vee}: H^{\prime \vee} \rightarrow H^{\vee}$ defines a right $R[x, f]$-homomorphism $\mathbf{D}\left(\mathbf{H}^{\prime}\right) \rightarrow \mathbf{D}(\mathbf{H})$, which we denote by $\mathbf{D}(\varphi)$.

1.13. Proposition. Let the situation and notation be as in Discussion 1.12 . There is a contravariant functor $\mathbf{D}:{ }_{R[x, f]} \operatorname{Mod} \rightarrow \operatorname{Mod}_{R[x, f]}$ which maps a left $R[x, f]$-module $(H, \alpha)$ to $\left(H^{\vee}, D(\alpha)\right)$ where $D(\alpha)$ is given by (11) in Discussion 1.12(i). 
1.14. Proposition. Let the situation and notation be as in Discussion 1.12 and let $\mathbf{H}=(H, \alpha)$ be a left $R[x, f]$-module. The right $R[x, f]$-module structures on $\mathbf{I}$ and $\mathbf{D}(\mathbf{H})=\left(\operatorname{Hom}_{l R}(H, I), D(\alpha)\right)$ are such that

$$
(m x)(h)=(m(x h)) x \quad \text { for all } m \in H^{\vee}=\operatorname{Hom}_{l R}(H, I) \text { and } h \in H .
$$

Proof. Recall that the right $R[x, f]$-module structure on $I$ is given by $\Psi:{ }_{f} I \rightarrow \operatorname{Hom}_{r}\left(R_{f}, I\right)$, so that $z x=(\Psi(z))(1)$ for all $z \in I$.

Let $m \in H^{\vee}=\operatorname{Hom}_{l R}(H, I)$ and $h \in H$. By (1) in Discussion 1.12(i), we have

$$
(m x)(h)=(D(\alpha)(m \otimes 1))(h)=\Psi(m(\alpha(1 \otimes h)))(1)=(m(\alpha(1 \otimes h))) x=(m(x h)) x .
$$

We now provide the right $R[x, f]$-module analogue of Discussion 1.12 ,

1.15. Discussion. The hypotheses and notation are as in Discussion 1.12 Let $\mathbf{M}=(M, \beta)$ be a right $R[x, f]$-module, where $\beta \in \operatorname{Hom}_{r R}\left(M \otimes_{R} R_{f}, M\right)$.

(i) Here we produce a left $R[x, f]$-module structure on $M^{\vee}$.

First apply the functor $(-)^{\vee}$ to the right $R$-homomorphism $\beta: M \otimes_{R} R_{f} \rightarrow M$ : the result is a left $R$-homomorphism $\beta^{\vee}: M^{\vee} \rightarrow \operatorname{Hom}_{r R}\left(M \otimes_{R} R_{f}, I\right)$. But there is an $(R, R)$-bimodule isomorphism $\left.\operatorname{Hom}_{r R}\left(M \otimes_{R} R_{f}, I\right)\right) \stackrel{\cong}{\longrightarrow} \operatorname{Hom}_{r R}\left(M, \operatorname{Hom}_{r R}\left(R_{f}, I\right)\right)$ given by Remark1.1(v), and use of the $(R, R)$-bimodule isomorphism $\Psi^{-1}$ produces a further $(R, R)$-bimodule isomorphism

$$
\operatorname{Hom}_{r R}\left(M, \operatorname{Hom}_{r R}\left(R_{f}, I\right)\right) \stackrel{\cong}{\longrightarrow} \operatorname{Hom}_{r R}\left(M, \operatorname{Hom}_{l R}\left(R_{f}, I\right)\right) .
$$

In addition, Remark 1.1(vi) provides an $(R, R)$-bimodule isomorphism

$$
\operatorname{Hom}_{r R}\left(M, \operatorname{Hom}_{l R}\left(R_{f}, I\right)\right) \stackrel{\cong}{\longrightarrow} \operatorname{Hom}_{l R}\left(R_{f}, \operatorname{Hom}_{r R}(M, I)\right) \text {. }
$$

Composition of these therefore yields a left $R$-homomorphism $\delta: M^{\vee} \longrightarrow \operatorname{Hom}_{l R}\left(R_{f}, M^{\vee}\right)$, and we shall denote by $D^{\prime}(\beta)$ the left $R$-homomorphism $R_{f} \otimes_{R} M^{\vee} \rightarrow M^{\vee}$ that corresponds to $\delta$ under the adjoint isomorphism of Remark 1.1(iv). (Note that $M^{\vee}=\operatorname{Hom}_{l R}(M, I)=$ $\operatorname{Hom}_{r R}(M, I)$.) Thus $D^{\prime}(\beta)$ makes $M^{\vee}$ into a left $R[x, f]$-module. We define

$$
\mathbf{D}^{\prime}(\mathbf{M})=\mathbf{D}(M, \beta):=\left(M^{\vee}, D^{\prime}(\beta)\right)=\left[M^{\vee}, \delta\right] .
$$

It is straightforward to use the above definition of $\delta$ to check that

(3) $\quad\left(D^{\prime}(\beta)(r \otimes h)\right)(m)=\left(\Psi^{-1}\left(r^{\prime} \mapsto h\left(\beta\left(m \otimes r^{\prime}\right)\right)\right)\right) r \quad$ for all $h \in M^{\vee}, r \in R_{f}$ and $m \in M$.

(ii) Now let $\mathbf{M}^{\prime}=\left(M^{\prime}, \beta^{\prime}\right)$ be a second right $R[x, f]$-module and let $\psi: \mathbf{M} \rightarrow \mathbf{M}^{\prime}$ be a right $R[x, f]$ homomorphism. An argument similar to that in Discussion[1.12(ii) shows that $\psi^{\vee}: M^{\prime \vee} \rightarrow M^{\vee}$ defines a left $R[x, f]$-homomorphism $\mathbf{D}^{\prime}\left(\mathbf{M}^{\prime}\right) \rightarrow \mathbf{D}^{\prime}(\mathbf{M})$, which we denote by $\mathbf{D}^{\prime}(\psi)$.

1.16. Proposition. Let the situation and notation be as in Discussion 1.15. There is a contravariant functor $\mathbf{D}^{\prime}: \operatorname{Mod}_{R[x, f]} \rightarrow R[x, f] \operatorname{Mod}$ which maps a right $R[x, f]$-module $(M, \beta)$ to $\left(M^{\vee}, D^{\prime}(\beta)\right)$ where $D^{\prime}(\beta)$ is given by (3) in Discussion 1.15)(i).

1.17. Proposition. Let the situation and notation be as in Discussion 1.15. Let $\mathbf{M}=(M, \beta)$ be a right $R[x, f]$-module, so that $\mathbf{D}^{\prime}(\mathbf{M})=\left(M^{\vee}, D^{\prime}(\beta)\right)$ is a left $R[x, f]$-module by Proposition 1.16 . The left action of $x$ on $M^{\vee}$ can be described as follows: for $h \in M^{\vee}$, the result xh of multiplying $h$ on the left by $x$ is the unique $h^{\prime} \in M^{\vee}$ for which

$$
\left(h^{\prime}(m)\right) r x=h(m r x) \quad \text { for all } m \in M \text { and } r \in R .
$$

Proof. First of all,

$$
\begin{aligned}
((x h)(m)) r x & =\left(\left(D^{\prime}(\beta)(1 \otimes h)\right)(m)\right) r x=\left(\Psi^{-1}\left(r^{\prime} \mapsto h\left(\beta\left(m \otimes r^{\prime}\right)\right)\right)\right) r x \\
& =\left(\Psi\left(\left(\Psi^{-1}\left(r^{\prime} \mapsto h\left(\beta\left(m \otimes r^{\prime}\right)\right)\right)\right) r\right)\right)(1)=\left(\left(r^{\prime} \mapsto h\left(\beta\left(m \otimes r^{\prime}\right)\right)\right) r\right)(1)=h(\beta(m \otimes r)) \\
& =h(\beta(m r \otimes 1))=h(m r x) .
\end{aligned}
$$

It therefore remains for us to show that if $h^{\prime} \in M^{\vee}$ is such that $\left(h^{\prime}(m)\right) r x=h(m r x)$ for all $m \in M$ and $r \in R$, then $h^{\prime}=x h$. It is therefore enough for us to show that if $h^{\prime \prime} \in M^{\vee}$ is such that $\left(h^{\prime \prime}(m)\right) r x=0$ 
for all $m \in M$ and $r \in R$, then $h^{\prime \prime}=0$. However, this is easy, because Lemma1.11 shows that $h^{\prime \prime}(m)=0$ for all $m \in M$.

Propositions 1.13 and 1.16 prepare the ground for several subsequent results in this paper.

1.18. Proposition. Let the situation and notation be as in Propositions 1.13 and 1.16, so that $R$ is $F$-finite and $I$ is an injective $R$-module with fixed $(R, R)$-bimodule isomorphism $\Psi:{ }_{f} I \rightarrow \operatorname{Hom}_{r}\left(R_{f}, I\right)$.

For each $R$-module $G$, we write $G^{\vee}=\operatorname{Hom}_{R}(G, I)$ as before. Let $\omega_{G}: G \longrightarrow\left(G^{\vee}\right)^{\vee}$ be the natural 'evaluation' R-homomorphism for which $\omega_{G}(g)(h)=h(g)$ for all $h \in G^{\vee}$ and $g \in G$. Recall that, as $G$ varies through the category ${ }_{R} \operatorname{Mod}$ of all $R$-modules and $R$-homomorphisms, the $\omega_{G}$ constitute a natural transformation from the identity functor on ${ }_{R} \operatorname{Mod}$ to the functor $\left((-)^{\vee}\right)^{\vee}$.

(i) If $\mathbf{H}=(H, \alpha)$ is a left $R[x, f]$-module, then $\omega_{H}$ is a left $R[x, f]$-module homomorphism from $\mathbf{H}$ to $\mathbf{D}^{\prime}(\mathbf{D}(\mathbf{H}))$. As $\mathbf{H}$ varies through ${ }_{R[x, f]}$ Mod, the $\omega_{H}$ constitute a natural transformation from the identity functor on that category to the functor $\mathbf{D}^{\prime} \circ \mathbf{D}$.

(ii) If $\mathbf{M}=(M, \beta)$ is a right $R[x, f]$-module, then $\omega_{M}$ is a right $R[x, f]$-module homomorphism from $\mathbf{M}$ to $\mathbf{D}\left(\mathbf{D}^{\prime}(\mathbf{M})\right)$. As $\mathbf{M}$ varies through $\operatorname{Mod}_{R[x, f]}$, the $\omega_{M}$ constitute a natural transformation from the identity functor on that category to the functor $\mathbf{D} \circ \mathbf{D}^{\prime}$.

Proof. (i) In view of Propositions 1.13 and 1.16, it only remains for us to show that, for a left $R[x, f]$ module $\mathbf{H}=(H, \alpha)$, the $R$-homomorphism $\omega_{H}: H \rightarrow\left(H^{\vee}\right)^{\vee}$ is actually a left $R[x, f]$-module homomorphism. To this end, we compare, for an $h \in H$, the elements $\omega_{H}(x h)$ and $x\left(\omega_{H}(h)\right)$. Now, $x \omega_{H}(h)$ is, by (4) in Proposition 1.17, the unique element $h^{\prime} \in\left(H^{\vee}\right)^{\vee}$ that satisfies

$$
\left(h^{\prime}(m)\right) r x=\omega_{H}(h)(m r x) \quad \text { for all } m \in H^{\vee} \text { and } r \in R .
$$

It is enough to show that $h^{\prime}=\omega_{H}(x h)$ satisfies this. But

$$
\left(\omega_{H}(x h)(m)\right) r x=(m(x h)) r x=(r(m(x h))) x=(m(r x h)) x=((m r)(x h)) x=(m r x)(h),
$$

where we have used (2) in 1.14 for the last equality. Since $(m r x)(h)=\omega_{H}(h)(m r x)$, the proof of part (i) is complete.

(ii) In view of Propositions 1.13 and 1.16, it only remains for us to show that, for a right $R[x, f]$ module $\mathbf{M}=(M, \beta)$, the $R$-homomorphism $\omega_{M}: M \rightarrow\left(M^{\vee}\right)^{\vee}$ is actually an $R[x, f]$-homomorphism. To this end, we compare, for an $m \in M$, the elements $\omega_{M}(m x)$ and $\left(\omega_{M}(m)\right) x$.

Now, for all $h \in M^{\vee}$, we have

$$
\begin{aligned}
\left(\left(\omega_{M}(m)\right) x\right)(h) & =\left(\omega_{M}(m)(x h)\right) x & & \text { (by (2) in 1.14) } \\
& =((x h)(m))) x & & \\
& =h(m x) & & \text { (by (4) in } 1.17 \text { ) } \\
& =\left(\omega_{M}(m x)\right)(h) . & &
\end{aligned}
$$

Hence $\omega_{M}(m x)=\left(\omega_{M}(m)\right) x$.

1.19. Remark. Let $R^{\prime}$ be a general commutative Noetherian ring and let $I$ be an injective $R^{\prime}$-module. For each $R^{\prime}$-module $M$ we write $M^{\vee}:=\operatorname{Hom}_{R^{\prime}}(M, I)$ and denote by $\omega_{M}$ the natural evaluation mapping $M \rightarrow\left(M^{\vee}\right)^{\vee}$ defined by $\omega_{M}(m)(h)=h(m)$ for all $h \in M^{\vee}$ and $m \in M$. We say that $M$ is $I$-reflexive if $\omega_{M}$ is an isomorphism. It is routine to check that, for an $R^{\prime}$-module $M$, the composition

$$
\left(\omega_{M}\right)^{\vee} \circ \omega_{M} \vee M^{\vee} \longrightarrow M^{\vee}
$$

is the identity map. Therefore, if $M$ is $I$-reflexive, then so too is $M^{\vee}$. It is easily verified that the full subcategory of $R^{\prime}$ Mod consisting of all $I$-reflexive modules is closed under finite direct sums, direct summands and extensions. But in general it is not a Serre subcategory of $R^{\prime}$ Mod, as can be seen by consideration of the case where $R^{\prime}$ is a Noetherian integral domain that is not a field and $I$ is taken to be the quotient field of $R^{\prime}$.

Suppose, in addition, that $\left(R^{\prime}, \mathfrak{m}\right)$ is (Noetherian) local and complete. Choose $I=E:=E_{R^{\prime}}\left(R^{\prime} / \mathfrak{m}\right)$, so that $(-)^{\vee}$ becomes the Matlis-duality functor $\operatorname{Hom}_{R^{\prime}}(-, E)$. In this case, E-reflexive modules are called Matlis-reflexive. It is well known that all Noetherian $R^{\prime}$-modules and all Artinian $R^{\prime}$-modules are Matlis-reflexive, and that $(-)^{\vee}$ provides a duality between the category of all Noetherian $R^{\prime}$-modules 
(and all $R^{\prime}$-homomorphisms between them) and the category of all Artinian $R^{\prime}$-modules (and all $R^{\prime}$ homomorphisms between them). Furthermore it was proved by E. Enochs [4, Proposition 1.3] that an $R^{\prime}$-module $M$ is Matlis-reflexive if and only if it can be embedded into a short exact sequence

$$
0 \longrightarrow N \longrightarrow M \longrightarrow A \longrightarrow 0
$$

in which $A$ is an Artinian $R^{\prime}$-module and $N$ is a Noetherian $R^{\prime}$-module. Therefore, the full subcategory of $R^{\prime}$ Mod consisting of all Matlis-reflexive modules is an Abelian category itself, and is actually the smallest Serre subcategory of $R^{\prime}$ Mod that contains all Noetherian modules and all Artinian modules.

Let the situation and notation be as in Proposition 1.18, so that $R$ is $F$-finite and $I$ is an injective $R$-module with a fixed $(R, R)$-bimodule isomorphism $\Psi:{ }_{f} I \rightarrow \operatorname{Hom}_{r R}\left(R_{f}, I\right)$. Let $\mathcal{L}_{I}$ be the category of all left $R[x, f]$-modules which are $I$-reflexive as $R$-modules, and all left $R[x, f]$-homomorphisms between them. Similarly let $\mathcal{R}_{I}$ be the category of right $R[x, f]$-modules which are $I$-reflexive as $R$-modules, and all right $R[x, f]$-homomorphisms between them.

In general, $\mathcal{L}_{I} \subseteq R[x, f]$ Mod and $\mathcal{R}_{I} \subseteq \operatorname{Mod}_{R[x, f]}$ are full subcategories, which are closed under finite direct sums, direct summands and extensions. If, in addition, $(R, \mathfrak{m})$ is local and complete and $I=E_{R}(R / \mathfrak{m})$, then $\mathcal{L}_{I}$ and $\mathcal{R}_{I}$ are Abelian categories by Remark 1.19

From the definitions of the functors $\mathbf{D}$ and $\mathbf{D}^{\prime}$, it is easy to use Remark 1.19 to see that they induce functors $\mathbf{D}: \mathcal{L}_{I} \rightarrow\left(\mathcal{R}_{I}\right)^{\mathrm{op}}$ and $\mathbf{D}^{\prime}: \mathcal{R}_{I} \rightarrow\left(\mathcal{L}_{I}\right)^{\mathrm{op}}$.

The following theorem is the main result of this paper.

1.20. Theorem. Let the situation and notation be as in 1.18 , so that $R$ is $F$-finite and $I$ is an injective $R$-module with a fixed $(R, R)$-bimodule isomorphism $\Psi:{ }_{f} I \rightarrow \operatorname{Hom}_{r R}\left(R_{f}, I\right)$. Then the functors $\mathbf{D}$ : $\mathcal{L}_{I} \rightarrow\left(\mathcal{R}_{I}\right)^{\mathrm{op}}$ and $\mathbf{D}^{\prime}: \mathcal{R}_{I} \rightarrow\left(\mathcal{L}_{I}\right)^{\mathrm{op}}$ are inverse equivalences of categories.

Proof. For any $\mathbf{H}=(H, \alpha) \in \mathcal{L}_{I}$, the evaluation mapping $\omega_{H}: H \rightarrow\left(H^{\vee}\right)^{\vee}$ is an $R[x, f]$-isomorphism, by Proposition 1.18(i). Therefore the natural transformation $\omega: \mathrm{Id} \rightarrow \mathbf{D}^{\prime} \circ \mathbf{D}$ of 1.18 (i) is a natural equivalence of functors on $\mathcal{L}_{I}$. Similarly, $\omega: \operatorname{Id} \rightarrow \mathbf{D} \circ \mathbf{D}^{\prime}$ is a natural equivalence of functors on $\mathcal{R}_{I}$.

From this theorem we have the following corollary in the complete local case.

1.21. Corollary. Assume that $(R, \mathfrak{m})$ is $F$-finite, complete and local, and let $I=E:=E_{R}(R / \mathfrak{m})$. We fix an $(R, R)$-bimodule isomorphism $\Psi:{ }_{f} E \rightarrow \operatorname{Hom}_{r R}\left(R_{f}, E\right)$.

(i) It follows from Theorem 1.20 that $\mathbf{D}$ and $\mathbf{D}^{\prime}$ are inverse equivalences between the category of left $R[x, f]$-modules that are Artinian as $R$-modules and the category of right $R[x, f]$-modules that are Noetherian as $R$-modules.

(ii) Similarly, $\mathbf{D}$ and $\mathbf{D}^{\prime}$ are inverse equivalences between the category of right $R[x, f]$-modules that are Artinian as $R$-modules and the category of left $R[x, f]$-modules that are Noetherian as $R$-modules.

\section{Graded annihilators}

Let $\mathfrak{B}$ be a subset of $R[x, f]$. It is easy to see that $\mathfrak{B}$ is a graded two-sided ideal of $R[x, f]$ if and only if there is an ascending chain $\left(\mathfrak{b}_{n}\right)_{n \in \mathbb{N}_{0}}$ of ideals of $R$ (which must, of course, be eventually stationary) such that $\mathfrak{B}=\bigoplus_{n \in \mathbb{N}_{0}} \mathfrak{b}_{n} x^{n}$. In particular, note that $R[x, f] x^{t}=\bigoplus_{i \geq t} R x^{i}$ is a graded two-sided ideal of $R[x, f]$, for each $t \in \mathbb{N}_{0}$.

2.1. Definitions. Let $\mathbf{H}=(H, \alpha)$ denote a left $R[x, f]$-module and let $\mathbf{M}=(M, \beta)$ denote a right $R[x, f]$-module; let $\mathfrak{B}$ be a two-sided ideal of $R[x, f]$.

The annihilator of $\mathbf{M}$ will be denoted by ann $\mathbf{M}_{R[x, f]}$. Thus

$$
\text { ann } \mathbf{M}_{R[x, f]}=\{\theta \in R[x, f] \mid g \theta=0 \text { for all } g \in M\},
$$

and this is a two-sided ideal of $R[x, f]$. The annihilator $\operatorname{ann}_{R[x, f]} \mathbf{H}$ of $\mathbf{H}$ is defined similarly; it is also a two-sided ideal of $R[x, f]$.

We define the graded annihilator gr-ann $\mathbf{M}_{R[x, f]}$ of the right $R[x, f]$-module $\mathbf{M}$ by

$$
\text { gr-ann } \mathbf{M}_{R[x, f]}=\left\{\sum_{i=0}^{n} r_{i} x^{i} \in R[x, f] \mid n \in \mathbb{N}_{0} \text { and } r_{i} \in R, r_{i} x^{i} \in \operatorname{ann} \mathbf{M}_{R[x, f]} \text { for all } i=0, \ldots, n\right\} \text {. }
$$


Thus gr-ann $\mathbf{M}_{R[x, f]}$ is the largest graded two-sided ideal of $R[x, f]$ contained in ann $\mathbf{M}_{R[x, f]}$.

The graded annihilator of $\mathbf{H}$ is defined similarly: it is the largest graded two-sided ideal of $R[x, f]$ that annihilates $\mathbf{H}$. See [18, 1.5]. Recall also that $\operatorname{ann}_{\mathbf{H}} \mathfrak{B}$ denotes the $R[x, f]$-submodule of $\mathbf{H}$ given by

$$
\operatorname{ann}_{\mathbf{H}} \mathfrak{B}=\{h \in \mathbf{H} \mid \theta h=0 \text { for all } \theta \in \mathfrak{B}\} .
$$

Observe that $M \mathfrak{B}$ is an $R[x, f]$-submodule of $\mathbf{M}$. For $t \in \mathbb{N}_{0}$, we have $M R[x, f] x^{t}=\left\{m x^{t}: m \in M\right\}$, and we shall therefore denote this $R[x, f]$-submodule of $\mathbf{M}$ by $\mathbf{M} x^{t}$. We shall say that $\mathbf{M}$ is $x$-divisible precisely when $\mathbf{M}=\mathbf{M} x$.

Recall (from [18, 1.2]) that $\mathbf{H}=(H, \alpha)$ is said to be $x$-torsion-free if $x h=0$, for $h \in H$, only when $h=0$. The set $\Gamma_{x}(H):=\left\{h \in H \mid x^{j} h=0\right.$ for some $\left.j \in \mathbb{N}\right\}$ is an $R[x, f]$-submodule of $\mathbf{H}$, called the $x$-torsion submodule of $\mathbf{H}$.

We are now going to compare, in the situation of Theorem 1.20, the graded annihilators of a left $R[x, f]$-module $\mathbf{H}$ and the right $R[x, f]$-module $\mathbf{D}(\mathbf{H})$, and also the graded annihilators of a right $R[x, f]$-module $\mathbf{M}$ and the left $R[x, f]$-module $\mathbf{D}^{\prime}(\mathbf{M})$.

Recall that an injective cogenerator for $R$ is an injective $R$-module $I$ such that $\operatorname{Hom}_{R}(G, I) \neq 0$ for every non-zero $R$-module $G$. See [22, p. 46]. It should be remarked that if $I$ is an injective cogenerator, then the evaluation map $\omega_{G}: G \longrightarrow\left(G^{\vee}\right)^{\vee}$ is a monomorphism for all $R$-modules $G$.

2.2. Proposition. Let the situation and notation be as in Theorem 1.20 , Let $\mathbf{H}=(H, \alpha)$ be a left $R[x, f]$-module and $\mathbf{M}=(M, \beta)$ be a right $R[x, f]$-module. Then

(i) $\operatorname{gr}^{-a n n n_{R[x, f]}} \mathbf{H} \subseteq \operatorname{gr}-\operatorname{ann} \mathbf{D}(\mathbf{H})_{R[x, f]}$;

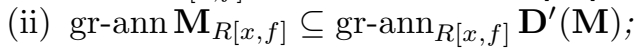

(iii) if $I$ is an injective cogenerator for $R$, we have

$$
\operatorname{gr-ann}_{R[x, f]} \mathbf{H}=\operatorname{gr-ann} \mathbf{D}(\mathbf{H})_{R[x, f]} \quad \text { and } \quad \operatorname{gr}-\operatorname{ann} \mathbf{M}_{R[x, f]}=\operatorname{gr-ann}_{R[x, f]} \mathbf{D}^{\prime}(\mathbf{M}) \text {; }
$$

(iv) in particular, in the special case in which $(R, \mathfrak{m})$ is local, and $I$ is taken to be $E_{R}(R / \mathfrak{m})$, we have

$$
\operatorname{gr-ann}_{R[x, f]} \mathbf{H}=\operatorname{gr-ann} \mathbf{D}(\mathbf{H})_{R[x, f]} \quad \text { and } \quad \operatorname{gr-ann} \mathbf{M}_{R[x, f]}=\operatorname{gr-ann}_{R[x, f]} \mathbf{D}^{\prime}(\mathbf{M}) .
$$

Proof. (i) Recall from (2) in 1.14 that the right action of $R[x, f]$ on $\mathbf{D}(\mathbf{H})=\left(H^{\vee}, D(\alpha)\right)$ is such that $(m x)(h)=(m(x h)) x$ for all $m \in H^{\vee}$ and all $h \in H$; an easy inductive argument shows that $\left(m x^{n}\right)(h)=\left(m\left(x^{n} h\right)\right) x^{n}$ for all $n \in \mathbb{N}$.

Now let $r \in R$ and $n \in \mathbb{N}_{0}$ be such that $r x^{n} H=0$. We show that $r x^{n}$ annihilates the right $R[x, f]$-module $\mathbf{D}(\mathbf{H})=\left(H^{\vee}, D(\alpha)\right)$. Let $m \in H^{\vee}$ and $h \in H$. Then, by the preceding paragraph,

$$
\left(m r x^{n}\right)(h)=\left((m r) x^{n}\right)(h)=\left((m r)\left(x^{n} h\right)\right) x^{n}=\left(m\left(r x^{n} h\right)\right) x^{n}=0 .
$$

It follows that gr-ann ${ }_{R[x, f]} \mathbf{H} \subseteq$ gr-ann $\mathbf{D}(\mathbf{H})_{R[x, f]}$.

(ii) Let $r \in R$ and $n \in \mathbb{N}_{0}$ be such that $M r x^{n}=0$. We show that $r x^{n}$ annihilates the left $R[x, f]$ module $\mathbf{D}^{\prime}(\mathbf{M})=\left(M^{\vee}, D(\beta)\right)$. This is clear when $n=0$, and so we suppose that $n>0$. Let $h \in M^{\vee}$ and $m \in M$. Then recall from (4) in 1.17 that $((x h)(m)) r^{\prime} x=h\left(m r^{\prime} x\right)$ for all $m \in M$ and $r^{\prime} \in R$. An easy inductive argument shows that

$$
\left(\left(x^{n} h\right)(m)\right) r^{\prime} x^{n}=h\left(m r^{\prime} x^{n}\right) \quad \text { for all } m \in M \text { and } r^{\prime} \in R .
$$

It follows from this that, for $r \in R$,

$$
\left(\left(r x^{n} h\right)(m)\right) r^{\prime} x^{n}=h\left(m r r^{\prime} x^{n}\right) \quad \text { for all } m \in M \text { and } r^{\prime} \in R .
$$

But, since $m r r^{\prime} x^{n}=m r^{\prime} r x^{n}=0$, we have $r x^{n} h(m)=0$ for all $m \in M$, by Lemma 1.11. Therefore $r x^{n} h=0$ and $r x^{n}$ annihilates the left $R[x, f]$-module $\mathbf{D}^{\prime}(\mathbf{M})$. Hence

$$
\text { gr-ann } \mathbf{M}_{R[x, f]} \subseteq \text { gr-ann }{ }_{R[x, f]} \mathbf{D}^{\prime}(\mathbf{M}) .
$$

(iii) By parts (i) and (ii), we have gr-ann ${ }_{R[x, f]} \mathbf{H} \subseteq \operatorname{gr-ann} \mathbf{D}(\mathbf{H})_{R[x, f]} \subseteq \operatorname{gr} \operatorname{ann}_{R[x, f]} \mathbf{D}^{\prime} \circ \mathbf{D}(\mathbf{H})$. However, since $I$ is an injective cogenerator for $R$, the homomorphism of left $R[x, f]$-modules $\omega_{H}$ : $H \longrightarrow\left(H^{\vee}\right)^{\vee}$ is actually an $R[x, f]$-monomorphism, and so it follows that

$$
\operatorname{gr-ann}_{R[x, f]} \mathbf{D}^{\prime} \circ \mathbf{D}(\mathbf{H}) \subseteq \operatorname{gr}^{-a n n n_{R[x, f]}} \mathbf{H} .
$$


The first equality is therefore proved. The second is proved similarly.

(iv) This is a special case of part (iii), because, when $(R, \mathfrak{m})$ is local, $E_{R}(R / \mathfrak{m})$ is an injective cogenerator for $R$.

2.3. Proposition. Let the situation and notation be as in 1.20 , and assume in addition that $(R, \mathfrak{m})$ is local and complete and that $I$ is taken to be $E:=E_{R}(R / \mathfrak{m})$. Let $\mathfrak{B}$ be a graded two-sided ideal of $R[x, f]$.

(i) Let $\mathbf{H}=(H, \alpha)$ be a left $R[x, f]$-module that is Matlis-reflexive as $R$-module. Let $\iota: \operatorname{ann}_{\mathbf{H}} \mathfrak{B} \longrightarrow$ $\mathbf{H}$ denote the inclusion $R[x, f]$-monomorphism. Then the induced homomorphism of right $R[x, f]$-modules $\mathbf{D}(\iota): \mathbf{D}(\mathbf{H}) \longrightarrow \mathbf{D}\left(\operatorname{ann}_{\mathbf{H}} \mathfrak{B}\right)$ has kernel $\mathbf{D}(\mathbf{H}) \mathfrak{B}$.

(ii) Let $\mathbf{M}=(M, \beta)$ be a right $R[x, f]$-module that is Noetherian as $R$-module. Let $\sigma: \mathbf{M B} \longrightarrow$ $\mathbf{M}$ denote the inclusion $R[x, f]$-monomorphism. Then the induced homomorphism of left $R[x, f]$-modules $\mathbf{D}^{\prime}(\sigma): \mathbf{D}^{\prime}(\mathbf{M}) \longrightarrow \mathbf{D}^{\prime}(\mathbf{M} \mathfrak{B})$ has kernel $\operatorname{ann}_{\mathbf{D}^{\prime}(\mathbf{M})} \mathfrak{B}$, so that $\operatorname{ann}_{\mathbf{D}^{\prime}(\mathbf{M})} \mathfrak{B} \cong$ $\mathbf{D}^{\prime}(\mathbf{M} / \mathbf{M B})$ as left $R[x, f]$-modules.

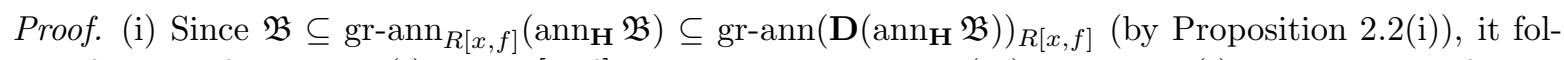
lows from the fact that $\mathbf{D}(\iota)$ is an $R[x, f]$-homomorphism that $\mathbf{D}(\mathbf{H}) \mathfrak{B} \subseteq \operatorname{Ker} \mathbf{D}(\iota)$. There is therefore an induced $R[x, f]$-epimorphism $\phi: \mathbf{D}(\mathbf{H}) / \mathbf{D}(\mathbf{H}) \mathfrak{B} \longrightarrow \mathbf{D}\left(\operatorname{ann}_{\mathbf{H}} \mathfrak{B}\right)$ for which $\phi(m+\mathbf{D}(\mathbf{H}) \mathfrak{B})=\mathbf{D}(\iota)(m)$ for all $m \in \mathbf{D}(\mathbf{H})$. Let $\lambda: \mathbf{D}(\mathbf{H}) \longrightarrow \mathbf{D}(\mathbf{H}) / \mathbf{D}(\mathbf{H}) \mathfrak{B}$ denote the canonical $R[x, f]$-epimorphism, and note that $\phi \circ \lambda=\mathbf{D}(\iota)$. We therefore have a commutative diagram

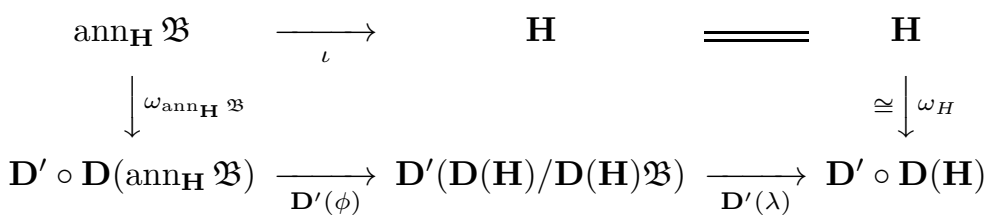

in the category ${ }_{R[x, f]}$ Mod. By Proposition 2.2(ii), we have $\operatorname{Im}^{\prime} \mathbf{D}^{\prime}(\lambda) \subseteq \operatorname{ann}_{\mathbf{D}^{\prime} \circ \mathbf{D}(\mathbf{H})} \mathfrak{B}$; since $\omega_{H}$ is an $R[x, f]$-isomorphism and $\mathbf{D}^{\prime}(\lambda)$ and $\mathbf{D}^{\prime}(\phi)$ are monomorphisms, it follows from the above commutative diagram that $\mathbf{D}^{\prime}(\phi)$ is an isomorphism. Since $I=E_{R}(R / \mathfrak{m})$ is an injective cogenerator for $R$, we can therefore deduce that $\phi$ is an isomorphism, so that $\mathbf{D}(\mathbf{H}) \mathfrak{B}=\operatorname{Ker} \mathbf{D}(\iota)$.

(ii) Let $j: \operatorname{ann}_{\mathbf{D}^{\prime}(\mathbf{M})} \mathfrak{B} \longrightarrow \mathbf{D}^{\prime}(\mathbf{M})$ denote the inclusion map and $k: \mathbf{M} \longrightarrow \mathbf{M} / \mathbf{M B}$ denote the natural epimorphism. Apply part (i) to the left $R[x, f]$-module $\mathbf{D}^{\prime}(\mathbf{M})$ to obtain an exact sequence

$$
0 \longrightarrow\left(\mathbf{D} \circ \mathbf{D}^{\prime}(\mathbf{M})\right) \mathfrak{B} \longrightarrow \mathbf{D} \circ \mathbf{D}^{\prime}(\mathbf{M}) \stackrel{\mathbf{D}(j)}{\longrightarrow} \mathbf{D}\left(\operatorname{ann}_{\mathbf{D}^{\prime}(\mathbf{M})} \mathfrak{B}\right) \longrightarrow 0
$$

in $\operatorname{Mod}_{R[x, f]}$. Since $M$ is Noetherian as $R$-module, the $R[x, f]$-homomorphism $\omega_{M}: \mathbf{M} \longrightarrow \mathbf{D}^{\prime} \circ \mathbf{D}(\mathbf{M})$ is an isomorphism. There is therefore a commutative diagram

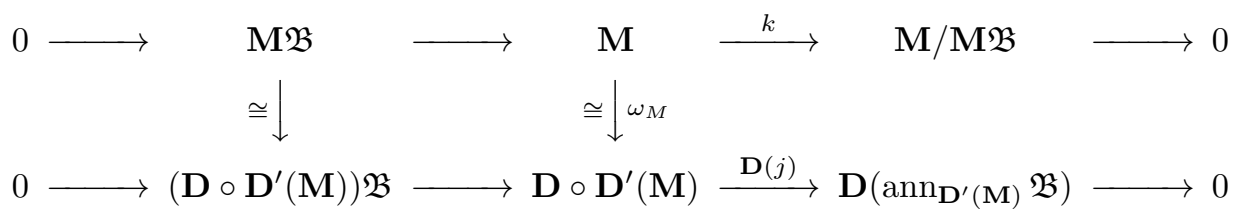

with exact rows in the category $\operatorname{Mod}_{R[x, f]}$. This induces an $R[x, f]$-isomorphism $\gamma: \mathbf{M} / \mathbf{M} \mathfrak{B} \cong$ $\mathbf{D}\left(\operatorname{ann}_{\mathbf{D}^{\prime}(\mathbf{M})} \mathfrak{B}\right)$ which, when inserted into the above diagram, is such that the extended diagram is still commutative. Now apply the functor $\mathbf{D}^{\prime}$ to the right-most square (involving $\gamma$ ) in that extended diagram: the result is the right-most square in the commutative diagram

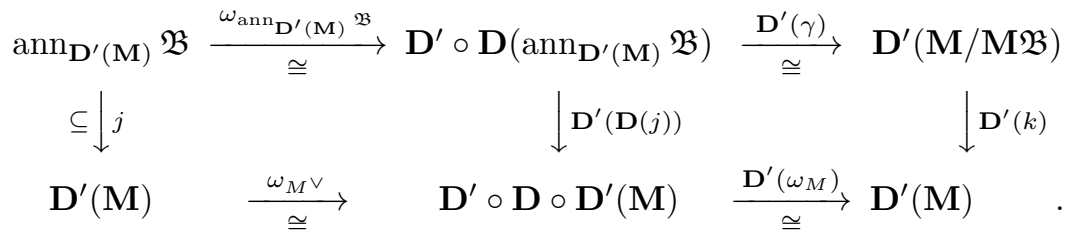

Note that $\mathbf{D}^{\prime}(\mathbf{M})$ is Artinian as $R$-module, so that $\omega_{M^{\vee}}$ and $\omega_{\mathrm{ann}_{\mathbf{D}^{\prime}(\mathbf{M})}(\mathfrak{B})}$ are both isomorphisms. Since $\mathbf{D}^{\prime}\left(\omega_{M}\right) \circ \omega_{M^{\vee}}=\operatorname{Id}_{M^{\vee}}$ (as noted in Remark 1.19), it follows from this commutative diagram that the 
kernel of the induced $R[x, f]$-homomorphism $\mathbf{D}^{\prime}(\sigma): \mathbf{D}^{\prime}(\mathbf{M}) \longrightarrow \mathbf{D}^{\prime}(\mathbf{M} \mathfrak{B})$, which is equal to the image of the $R[x, f]$-homomorphism $\mathbf{D}^{\prime}(k): \mathbf{D}^{\prime}(\mathbf{M} / \mathbf{M} \mathfrak{B}) \longrightarrow \mathbf{D}^{\prime}(\mathbf{M})$, is precisely ann $\mathbf{D}_{\mathbf{D}^{\prime}(\mathbf{M})} \mathfrak{B}$.

2.4. Corollary. Let the situation and notation be as in 1.20 , and assume in addition that $(R, \mathfrak{m})$ is local and complete and that $I$ is taken to be $E:=E_{R}(R / \mathfrak{m})$. Let $\mathbf{M}$ be a right $R[x, f]$-module that is Noetherian as $R$-module. Then $\mathbf{M}$ is x-divisible if and only if $\mathbf{D}^{\prime}(\mathbf{M})$ is x-torsion-free.

Proof. Let $j: \mathbf{M} x \longrightarrow \mathbf{M}$ be the inclusion $R[x, f]$-monomorphism. By Proposition 2.3(ii), the kernel of $\mathbf{D}^{\prime}(j): \mathbf{D}^{\prime}(\mathbf{M}) \longrightarrow \mathbf{D}^{\prime}(\mathbf{M} x)$ is $\operatorname{ann}_{\mathbf{D}^{\prime}(\mathbf{M})} R[x, f] x$.

Now $\mathbf{M}$ is $x$-divisible if and only if $j$ is an isomorphism; since $E$ is an injective cogenerator for $R$, this is the case if and only if $\mathbf{D}^{\prime}(j)$ is an isomorphism; and, by the above comment (and the fact that $\mathbf{D}^{\prime}(j)$ must always be an $R[x, f]$-epimorphism), $\mathbf{D}^{\prime}(j)$ is an isomorphism if and only if $\operatorname{ann}_{\mathbf{D}^{\prime}(\mathbf{M})} R[x, f] x=0$, that is, if and only if $\mathbf{D}^{\prime}(\mathbf{M})$ is $x$-torsion-free.

\section{SOME APPLICATIONS}

As was mentioned in the Introduction, the Hartshorne-Speiser-Lyubeznik Theorem has been applied to establish the existence of a uniform Frobenius test exponent for Frobenius closures of parameter ideals in a local ring $(R, \mathfrak{m})$ that is Cohen-Macaulay, or just generalized Cohen-Macaulay. The non-local version of the Hartshorne-Speiser-Lyubeznik Theorem given in [17, Corollary 1.8] can be reformulated as follows: if ( $R$ is not necessarily local and) $H$ is a left $R[x, f]$-module that is Artinian as $R$-module, then there exists an $e \in \mathbb{N}_{0}$ such that $\operatorname{ann}_{H} R[x, f] x^{e}=\operatorname{ann}_{H} R[x, f] x^{e+1}$. With this in mind, one can regard the following result as a 'dual Hartshorne-Speiser-Lyubeznik Theorem' for the case where $(R, \mathfrak{m})$ is $F$-finite, local and complete.

3.1. Theorem. Assume that $(R, \mathfrak{m})$ is F-finite, local and complete. Let $\mathbf{M}=(M, \beta)$ be a right $R[x, f]$ module that is Noetherian as $R$-module. Then there exists $e \in \mathbb{N}_{0}$ such that $\mathbf{M} x^{e}=\mathbf{M} x^{e+1}$, that is, such that $M R[x, f] x^{e}=M R[x, f] x^{e+1}$.

Proof. Let $E:=E_{R}(R / \mathfrak{m})$. Select an $(R, R)$-bimodule isomorphism $\Psi:{ }_{f} E \stackrel{\cong}{\longrightarrow} \operatorname{Hom}_{r R}\left(R_{f}, E\right)$ : recall that Lemma 1.4 ensures that there is such a $\Psi$. By Proposition 1.16 and Remark 1.19, we know that $\mathbf{D}^{\prime}(\mathbf{M})=\left(M^{\vee}, D(\beta)\right)$ is a left $R[x, f]$-module; by Matlis duality, as $R$-module, $M^{\vee}=\operatorname{Hom}_{R}(M, E)$ is Artinian. Therefore, by the Hartshorne-Speiser-Lyubeznik Theorem, there exists an $e \in \mathbb{N}_{0}$ such that $\operatorname{ann}_{\mathbf{D}^{\prime}(\mathbf{M})} R[x, f] x^{e}=\operatorname{ann}_{\mathbf{D}^{\prime}(\mathbf{M})} R[x, f] x^{e+1}$.

Let $i: \mathbf{M} x^{e} \stackrel{\subseteq}{\longrightarrow} \mathbf{M}, j: \mathbf{M} x^{e+1} \stackrel{\subseteq}{\longrightarrow} \mathbf{M}$ and $k: \mathbf{M} x^{e+1} \stackrel{\subseteq}{\longrightarrow} \mathbf{M} x^{e}$ be the inclusion $R[x, f]$ homomorphisms, so that $i \circ k=j$. In view of Proposition 2.3(ii), there is a commutative diagram

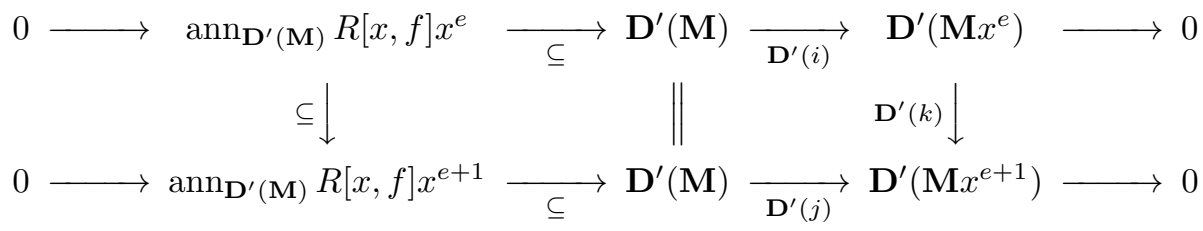

with exact rows in the category $R[x, f]$ Mod. Since $\operatorname{ann}_{\mathbf{D}^{\prime}(\mathbf{M})} R[x, f] x^{e}=\operatorname{ann}_{\mathbf{D}^{\prime}(\mathbf{M})} R[x, f] x^{e+1}$, we see that $\mathbf{D}^{\prime}(k)$ must be an isomorphism; therefore, $k$ must be an isomorphism, because $E$ is an injective cogenerator for $R$. Therefore $\mathbf{M} x^{e}=\mathbf{M} x^{e+1}$.

It is natural to ask whether the conclusion of Theorem 3.1 is still valid if we drop the assumptions about $R$ (except the one that $R$ has characteristic $p$ ). In Theorem 3.4 below, we shall show that this is indeed the case. We first present two preparatory lemmas, in which we assume only that $R$ is a commutative Noetherian ring of characteristic $p$.

3.2. Lemma. Let $\mathbf{M}=(M, \beta)$ be a right $R[x, f]$-module. Suppose that there is an element $s \in R$ such that $M s \subseteq M x$. Then, for all $k \in \mathbb{N}$, we have $M s^{2} \subseteq M x^{k}$.

Proof. We prove the lemma by induction on $k$. When $k=1$, there is nothing to prove. Assume that $M s^{2} \subseteq M x^{k}$ for a $k \in \mathbb{N}$. Then, since $M s^{p} \subseteq M s^{2} \subseteq M x^{k}$, we have $M s^{p} x \subseteq M x^{k+1}$. Thus $M s^{2}=(M s) s \subseteq(M x) s=M s^{p} x \subseteq M x^{k+1}$. The lemma is therefore proved by induction. 
3.3. Lemma. Let $\mathbf{M}=(M, \beta)$ be a right $R[x, f]$-module and $S$ be a multiplicatively closed subset of $R$. Then the module of fractions $S^{-1} M$ has a natural right $\left(S^{-1} R\right)[x, f]$-module structure in which

$$
\left(\frac{m}{s}\right) x=\frac{m s^{p-1} x}{s} \quad \text { for all } m \in M \text { and } s \in S .
$$

This structure is such that $S^{-1}\left(M x^{k}\right)=\left(S^{-1} M\right) x^{k}$ for all $k \in \mathbb{N}$.

Proof. It is straightforward to construct a right $\left(S^{-1} R\right)[x, f]$-module structure on $S^{-1} M$ with the specified properties. An easy inductive argument shows that

$$
\left(\frac{m}{s}\right) x^{k}=\frac{m s^{p^{k}-1} x^{k}}{s} \text { for all } k \in \mathbb{N}, m \in M \text { and } s \in S .
$$

It is clear from this that $S^{-1}\left(M x^{k}\right) \supseteq\left(S^{-1} M\right) x^{k}$ for a $k \in \mathbb{N}$.

To establish the reverse inclusion, let $\alpha \in S^{-1}\left(M x^{k}\right)$, so that $\alpha=\left(m x^{k}\right) / s$ for some $m \in M$ and $s \in S$. Then

$$
\alpha=\frac{m x^{k}}{s}=\frac{m x^{k} s^{p^{k}-1}}{s^{p^{k}}}=\frac{m\left(s^{p^{k}-1}\right)^{p^{k}} x^{k}}{s^{p^{k}}}=\frac{m\left(s^{p^{k}}\right)^{p^{k}-1} x^{k}}{s^{p^{k}}}=\left(\frac{m}{s^{p^{k}}}\right) x^{k} \in\left(S^{-1} M\right) x^{k} .
$$

3.4. Theorem. Assume only that $R$ is a commutative Noetherian ring of characteristic $p$. Let $\mathbf{M}=$ $(M, \alpha)$ be a right $R[x, f]$-module that is Noetherian as $R$-module. Then there exists $k \in \mathbb{N}_{0}$ such that $\mathbf{M} x^{k}=\mathbf{M} x^{k+1}$.

Proof. It is straightforward to check that $\left(0:_{M} R x^{k}\right):=\left\{m \in M \mid m R x^{k}=0\right\}$ is an $R[x, f]$-submodule of $M$ for each $k \in \mathbb{N}$. Define $\left(0:_{M} R x^{\infty}\right):=\bigcup_{k \in \mathbb{N}}\left(0:_{M} R x^{k}\right)$; this is also an $R[x, f]$-submodule of $M$; therefore $M^{\gamma}:=M /\left(0:_{M} R x^{\infty}\right)$ is again a right $R[x, f]$-module.

Since $M$ is Noetherian as $R$-module, the ascending chain

$$
\left(0:_{M} R x\right) \subseteq\left(0:_{M} R x^{2}\right) \subseteq \cdots \subseteq\left(0:_{M} R x^{k}\right) \subseteq \cdots
$$

must eventually be stationary, say at $\left(0:_{M} R x^{\ell}\right)$; then $\left(0:_{M} R x^{\infty}\right)=\left(0:_{M} R x^{\ell}\right)$. We point out that if there exists $k \in \mathbb{N}_{0}$ such that $M^{\gamma} x^{k}=M^{\gamma} x^{k+1}$, then $M x^{k} \subseteq M x^{k+1}+\left(0:_{M} R x^{\ell}\right)$, so that multiplication on the right by $x^{\ell}$ yields that $M x^{k+\ell} \subseteq M x^{k+\ell+1}$. Thus, if the conclusion of the theorem is true for $M^{\gamma}$, then it is true for $M$.

Next, we define $M \cdot x^{\infty}:=\bigcap_{k \in \mathbb{N}} M x^{k}$; this is an $R[x, f]$-submodule of $M$, and so $M^{\sigma}:=M / M \cdot x^{\infty}$ is again a right $R[x, f]$-module. Note also that, if there exists $k \in \mathbb{N}_{0}$ such that $M^{\sigma} x^{k}=M^{\sigma} x^{k+1}$, then $M x^{k} \subseteq M x^{k+1}+M \cdot x^{\infty}=M x^{k+1}$. Thus, if the conclusion of the theorem is true for $M^{\sigma}$, then it is true for $M$.

Consider the sequence of right $R[x, f]$-modules

$$
M \rightarrow M^{\sigma} \rightarrow\left(M^{\sigma}\right)^{\gamma} \rightarrow\left(\left(M^{\sigma}\right)^{\gamma}\right)^{\sigma} \rightarrow\left(\left(\left(M^{\sigma}\right)^{\gamma}\right)^{\sigma}\right)^{\gamma} \rightarrow \cdots,
$$

where, at each stage, the arrow denotes the appropriate natural $R[x, f]$-epimorphism. The first three paragraphs of this proof show that, if the claim in the theorem holds for any module $M^{\prime}$ in this sequence, then it holds for all modules to the left of $M^{\prime}$, including $M$ itself. If, for each $n \in \mathbb{N}$, we let $K_{n}$ denote the kernel of the composition of the first $n$ epimorphisms in this sequence, then $K_{1} \subseteq K_{2} \subseteq \cdots \subseteq K_{n} \subseteq \cdots$ is an ascending chain of $R$-submodules of $M$, and therefore eventually stationary. This means that, in the above displayed sequence, there is a term to the right of which all the epimorphisms are isomorphisms. Therefore it is enough for us to prove the theorem under the additional assumptions that

$$
\left(0:_{M} R x^{\infty}\right)=0 \quad \text { and } M \cdot x^{\infty}=0 .
$$

We shall show now that these additional assumptions force $M$ to be zero (in which case $M x=$ $\left.M x^{2}\right)$. Suppose that $M \neq 0$, and seek a contradiction. Denote by $\operatorname{Min}_{R}(M)$ the set of minimal prime ideals in $\operatorname{Supp}_{R}(M)$. This is a non-empty set, because $M \neq 0$. We set $S:=R \backslash \bigcup_{\mathfrak{p} \in \operatorname{Min}_{R}(M)} \mathfrak{p}$, a multiplicatively closed subset of $R$. Then, the $S^{-1} R$-module $S^{-1} M$ is a non-trivial Artinian module, since $\operatorname{dim}_{S^{-1} R} S^{-1} M=0$. By Lemma 3.3, $S^{-1} M$ has a natural structure as a right $\left(S^{-1} R\right)[x, f]$ module. Consider the descending sequence of right $\left(S^{-1} R\right)[x, f]$-submodules

$$
S^{-1} M \supseteq\left(S^{-1} M\right) x \supseteq\left(S^{-1} M\right) x^{2} \supseteq\left(S^{-1} M\right) x^{3} \supseteq\left(S^{-1} M\right) x^{4} \supseteq \cdots
$$


of $S^{-1} M$. Since $S^{-1} M$ is Artinian as $S^{-1} R$-module, we must have $\left(S^{-1} M\right) x^{k}=\left(S^{-1} M\right) x^{k+1}$ for some $k \in \mathbb{N}$. Then, $S^{-1}\left(M x^{k}\right)=S^{-1}\left(M x^{k+1}\right)$ by virtue of Lemma 3.3. Since $M x^{k}$ is finitely generated as $R$ module, it follows that there is an element $s \in S$ such that $\left(M x^{k}\right) s \subseteq M x^{k+1}$. Now, applying Lemma 3.2 to the right $R[x, f]$-module $M x^{k}$, we have that $\left(M x^{k}\right) s^{2} \subseteq M x^{k+k^{\prime}}$ for all $k^{\prime} \in \mathbb{N}$. Therefore $\left(M x^{k}\right) s^{2} \subseteq M \cdot x^{\infty}=0$ by the assumption (5). Since $\left(M x^{k}\right) s^{2}=M s^{2 p^{k}} x^{k}$, we have shown that $M s^{2 p^{k}} x^{k}=0$; hence $M s^{2 p^{k}} \subseteq\left(0:_{M} R x^{k}\right) \subseteq\left(0:_{M} R x^{\infty}\right)=0$. Consequently we have $M s^{2 p^{k}}=0$. Therefore $s$ belongs to $\sqrt{\operatorname{ann}_{R}(M)}$; therefore $s \in \mathfrak{p}$ for all $\mathfrak{p} \in \operatorname{Min}_{R}(M)$. But this contradicts the fact that $s$ is an element of $S$.

One of the main results of [18] is that, if $\mathbf{H}$ is an $x$-torsion-free left $R[x, f]$-module that is Artinian as $R$-module, then there are only finitely many graded annihilators of $R[x, f]$-submodules of $\mathbf{H}$. See [18. Corollary 3.11]. This result has relevance to the existence of tight closure test elements in certain circumstances: see [18, Corollary 4.7] and [20, Theorem 3.5]. We can use our work in $\S 1$ and $\S 2$ to obtain a dual result in the special case where $R$ is $F$-finite, local and complete.

3.5. Theorem. Assume that $(R, \mathfrak{m})$ is $F$-finite, local and complete. Let $\mathbf{M}=(M, \beta)$ be an $x$-divisible right $R[x, f]$-module that is Noetherian as $R$-module. Then there are only finitely many graded annihilators of $R[x, f]$-homomorphic images of $\mathbf{M}$.

Proof. Select an $(R, R)$-bimodule isomorphism $\Psi:{ }_{f} E_{R}(R / \mathfrak{m}) \stackrel{\cong}{\longrightarrow} \operatorname{Hom}_{R}\left(R_{f}, E_{R}(R / \mathfrak{m})\right)$ : recall that Lemma 1.4 ensures that there is such a $\Psi$. Use the notation of 1.20 , but take $I$ to be $E:=E_{R}(R / \mathfrak{m})$. By Proposition 1.16, we know that $\mathbf{D}^{\prime}(\mathbf{M})$ is a left $R[x, f]$-module; as such, it is $x$-torsion-free, by Corollary 2.4. By Matlis duality, as $R$-module, $M^{\vee}=\operatorname{Hom}_{R}(M, E)$ is Artinian. By [18, Lemma 1.9, Definition 1.10 and Corollary 3.11], there are only finitely many graded annihilators of $R[x, f]$-submodules of $\mathbf{D}^{\prime}(\mathbf{M})$. It is therefore enough for us to show that, if $\mathfrak{B}$ is the graded-annihilator of some $R[x, f]$ homomorphic image of $\mathbf{M}$, then $\mathfrak{B}$ is the graded-annihilator of some $R[x, f]$-submodule of $\mathbf{D}^{\prime}(\mathbf{M})$. This we do.

Thus there is an $R[x, f]$-submodule $\mathbf{L}$ of $\mathbf{M}$ such that $\mathfrak{B}=\operatorname{gr}$-ann $(\mathbf{M} / \mathbf{L})_{R[x, f]}$. Therefore $\mathbf{M} \mathfrak{B} \subseteq \mathbf{L}$, so that there is an $R[x, f]$-epimorphism $\mathbf{M} / \mathbf{M} \mathfrak{B} \longrightarrow \mathbf{M} / \mathbf{L}$. Therefore

$$
\mathfrak{B} \subseteq \operatorname{gr}-\operatorname{ann}(\mathbf{M} / \mathbf{M} \mathfrak{B})_{R[x, f]} \subseteq \operatorname{gr}-\operatorname{ann}(\mathbf{M} / \mathbf{L})_{R[x, f]}=\mathfrak{B},
$$

so that $\mathfrak{B}=\operatorname{gr-ann}(\mathbf{M} / \mathbf{M} \mathfrak{B})_{R[x, f]}$. It now follows from Proposition 2.2(iv) that

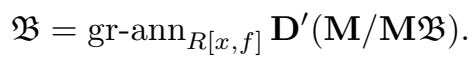

However, Proposition 2.3)(ii) shows that there is an isomorphism $\mathbf{D}^{\prime}(\mathbf{M} / \mathbf{M} \mathfrak{B}) \cong \operatorname{ann}_{\mathbf{D}^{\prime}(\mathbf{M})} \mathfrak{B}$ of left $R[x, f]$-modules; therefore $\mathfrak{B}$ is the graded annihilator of the $R[x, f]$-submodule $\operatorname{ann}_{\mathbf{D}^{\prime}(\mathbf{M})} \mathfrak{B}$ of $\mathbf{D}^{\prime}(\mathbf{M})$. This completes the proof.

It is natural to ask whether the conclusion of Theorem 3.5 is still valid if we drop the assumptions about $R$ (except the one that $R$ has characteristic $p$ ).

3.6. Question. Assume only that $R$ is (a commutative Noetherian ring) of characteristic $p$. Let $\mathbf{M}$ be an $x$-divisible right $R[x, f]$-module that is Noetherian as $R$-module. Is the set of graded annihilators of $R[x, f]$-homomorphic images of $\mathbf{M}$ finite?

At the time of writing, we are not able to answer Question 3.6.

\section{REFERENCES}

[1] M. Blickle and G. Boeckle, Cartier modules: finiteness results, J. reine angew. Math., to appear; arXiv math.AC 0909.2531v1.

[2] F. Enescu, F-injective rings and F-stable primes, Proc. Amer. Math. Soc. 131 (2003) 3379-3386.

[3] F. Enescu, Local cohomology and F-stability, J. Algebra 322 (2009) 3063-3077.

[4] E. Enochs, Flat Covers and Flat Cotorsion Modules, Proc. Amer. Math. Soc., 92 (1984) 179-184.

[5] R. Fedder, F-purity and rational singularity in graded complete intersection rings, Transactions Amer. Math. Soc. 301 (1987) 47-62.

[6] R. Fedder and K-i. Watanabe, A characterization of F-regularity in terms of F-purity, in: M. Hochster, C. Huneke and J. D. Sally (Eds.), Commutative algebra: proceedings of a microprogram held June 15 - July 2, 1987, Mathematical Sciences Research Institute Publications 15, Springer, New York, 1989, pp. 227-245. 
[7] O. Gabber, Notes on some t-structures, Geometric aspects of Dwork theory, Vol. I,II, Walter de Gruyter GmbH \& Co. KG, Berlin, 2004, pp. 711-734.

[8] N. Hara and K-i. Watanabe, The injectivity of Frobenius acting on cohomology and local cohomology modules, Manuscripta Math. 90 (1996) 301-315.

[9] R. Hartshorne and R. Speiser, Local cohomological dimension in characteristic p, Annals of Math. 105 (1977) 45-79.

[10] M. Hochster and J. L. Roberts, The purity of the Frobenius and local cohomology, Advances in Math. 21 (1976) $117-172$.

[11] C. Huneke, M. Katzman, R. Y. Sharp and Y. Yao, Frobenius test exponents for parameter ideals in generalized Cohen-Macaulay local rings, J. Algebra 305 (2006) 516-539.

[12] M. Katzman, Parameter-test-ideals of Cohen-Macaulay rings, Compositio Math. 144 (2008) 933-948.

[13] M. Katzman and R. Y. Sharp, Uniform behaviour of the Frobenius closures of ideals generated by regular sequences, J. Algebra 295 (2006) 231-246.

[14] G. Lyubeznik, F-modules: applications to local cohomology and D-modules in characteristic $p>0$, J. reine angew. Math. 491 (1997) 65-130.

[15] H. Matsumura, Commutative ring theory, Cambridge Studies in Advanced Mathematics 8, Cambridge University Press, 1986.

[16] J. J. Rotman, Advanced modern algebra, Prentice Hall, Inc., Upper Saddle River, NJ, 2002.

[17] R. Y. Sharp, Tight closure test exponents for certain parameter ideals, Michigan Math. J. 54 (2006) 307-317.

[18] R. Y. Sharp, Graded annihilators of modules over the Frobenius skew polynomial ring, and tight closure, Transactions Amer. Math. Soc. 359 (2007) 4237-4258.

[19] R.Y.Sharp, On the Hartshorne-Speiser-Lyubeznik Theorem about Artinian modules with a Frobenius action, Proc. Amer. Math. Soc. 135 (2007) 665-670.

[20] R. Y. Sharp, Graded annihilators and tight closure test ideals, J. Algebra 322 (2009) 3410-3426.

[21] R. Y. Sharp, An excellent F-pure ring of prime characteristic has a big tight closure test element, Transactions Amer. Math. Soc., to appear; arXiv math.AC 0907.2857.

[22] D. W. Sharpe and P. Vámos, Injective modules, Cambridge Tracts in Mathematics and Mathematical Physics 62 Cambridge University Press, 1972.

[23] K. E. Smith, Tight closure of parameter ideals, Inventiones mathematicae 115 (1994) 41-60.

[24] Y. Yoshino, Skew-polynomial rings of Frobenius type and the theory of tight closure, Communications in Algebra 22 (1994) 2473-2502.

Department of Pure Mathematics, University of Sheffield, Hicks Building, Sheffield S3 7RH, United KINGDOM

E-mail address: R.Y.Sharp@sheffield.ac.uk

Department of Mathematics, Faculty of Science, Okayama University, Tsushima-Naka 3-1-1, Okayama 7008530, JAPAN

E-mail address: yoshino@math.okayama-u.ac.jp 